\title{
Dynamical analysis of generalized Galileon cosmology
}

\author{
Genly Leon ${ }^{a, b}$ and Emmanuel N. Saridakis ${ }^{c, d}$ \\ ${ }^{a}$ Departamento de Matemática, Universidad Central de Las Villas, Santa Clara CP 54830, Cuba \\ ${ }^{b}$ Instituto de Física, Pontificia Universidad Católica de Valparaíso, Casilla 4950, Valparaíso, Chile \\ ${ }^{c}$ Physics Division, National Technical University of Athens, 15780 Zografou Campus, Athens, \\ Greece \\ ${ }^{d}$ CASPER, Physics Department, Baylor University, Waco, TX 76798-7310, USA \\ E-mail: genly.leon@ucv.cl, Emmanuel_Saridakis@baylor.edu
}

\begin{abstract}
We perform a detailed dynamical analysis of generalized Galileon cosmology, incorporating also the requirements of ghost and instabilities absence. We find that there are not any new stable late-time solutions apart from those of standard quintessence. Furthermore, depending on the model parameters the Galileons may survive at late times or they may completely disappear by the dynamics, however the corresponding observables are always independent of the Galileon terms, determined only by the usual action terms. Thus, although the Galileons can play an important role at inflationary or at recent times, in the future, when the universe will asymptotically reach its stable state, they will not have any effect on its evolution.
\end{abstract}

KEYWORDS: Galileon cosmology, dark energy, dynamical analysis 


\section{Contents}

1 Introduction 1

2 Generalized Galileon cosmology 3

3 Phase space analysis $\quad 6$

3.1 Scenario 1: Exponential potential and exponential coupling function $\quad 7$

$\begin{array}{lll}3.1 .1 & \text { Finite phase-space analysis } & 9\end{array}$

$\begin{array}{ll}3.1 .2 & \text { Phase-space analysis at infinity }\end{array}$

3.2 Scenario 2: Exponential potential and power-law coupling function 11

$\begin{array}{lll}3.2 .1 & \text { Finite phase-space analysis } & 13\end{array}$

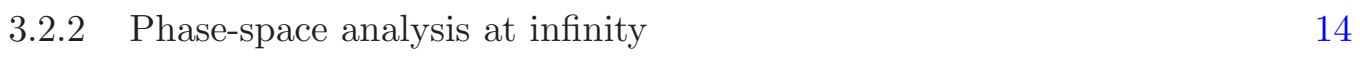

4 Cosmological Implications $\quad \mathbf{1 6}$

4.1 Scenario 1: Exponential potential and exponential coupling function $\quad 16$

4.2 Scenario 2: Exponential potential and power-law coupling function 20

5 Conclusions $\quad 22$

$\begin{array}{ll}\text { A Stability of Scenario } 1 & \mathbf{2 4}\end{array}$

A.1 Stability of the finite critical points 24

$\begin{array}{lll}\text { A.2 Stability of the critical points at infinity } & 25\end{array}$

B Stability of Scenario 2 26

B.1 Stability of the finite critical points 26

B.2 Stability of the critical points at infinity 30

\section{Introduction}

There are two approaches one can follow in order to describe the observed universe acceleration. The first is to introduce the concept of dark energy, usually adding extra scalar fields (see [1] and references therein) in the right-hand-side of the field equations of General Relativity, while the second is to modify the left-hand-side of the general relativistic field equations, that is to modify the gravitational theory itself (see $[2,3]$ and references therein).

A recently re-discovered, very general class of scalar-field theories is based on the introduction of higher derivatives in the action, with the requirement of maintaining the equations of motion second order. Although the most general second-order theories avoiding the Ostrogradsky instabilities [4] were already derived in [5], a particular class, dubbed 
Galileon, was constructed in [6] for the Minkowski metric and in [7, 8] for dynamical geometries, while in [9] the results of [5] were re-discovered in the context of (extended) Galileon framework. In this formalism the Lagrangian is suitably constructed in order for the field equations to be invariant under the Galilean symmetry $\phi \rightarrow \phi+c, \partial_{\mu} \phi \rightarrow \partial_{\mu} \phi+b_{\mu}$ in the limit of Minkowski spacetime, with $c, b_{\mu}$ constants. The four-dimensional Lagrangian that preserves these symmetries contains five unique terms consisting of scalar combinations of $\partial_{\mu} \phi, \partial_{\mu} \partial_{\nu} \phi$ and $\square \phi$ (since General Relativity does not accept the satisfaction of Galilean symmetry in curved spacetime, higher derivatives are necessary in the action [10-12]), and the corresponding couplings are not renormalized by loop corrections [13]. Furthermore, a significant advantage is that the derivative self-couplings of the scalar screen the deviations from General Relativity at high gradient regions (small scales or high densities) through the Vainshtein mechanism [14], thus satisfying solar system and early universe constraints [15-17].

Application of the above construction in cosmological frameworks gives rise to the Galileon cosmology, which proves to be very interesting and has been investigated in detail in the literature. In particular, one can investigate the universe evolution and late-time acceleration [18-27], inflation [28-35] and non-Gaussianities [36-40], the reheating of the post-inflationary universe [41], the growth history [42-44], the cosmological bounce [45-47], the cosmological perturbations [48-52], the spherical solutions [53-58], the stability issues [59-64], and also he can use observational data in order to constrain the parameters of the theory [65-73]. Moreover, one can study and extend the properties of Galileon theory itself [74-94], and examine the relation of Galileons with other frameworks [95-106].

Since Galileon cosmology exhibits interesting phenomenological features, in the present work we perform a phase-space and stability analysis of such a scenario, investigating in a systematic way the possible cosmological behaviors, focusing on the late-time stable solutions. Such an approach allows us to bypass the high non-linearities of the cosmological equations, which prevent any complete analytical treatment, obtaining a qualitative description of the global dynamics of these models, which is independent of the initial conditions and the specific evolution of the universe. Moreover, in these asymptotic solutions we calculate various observable quantities, such as the dark energy density and equation-of-state parameters and the deceleration parameter, and in order to ensure that these solutions are free of ghosts and Laplacian instabilities we additionally calculate the relevant perturbations quantities. Interestingly enough, our analysis shows that Galileon cosmology does not exhibit any new stable late-time solutions apart from those of standard quintessence, and moreover the corresponding observables are always independent of the Galileon terms. Thus, although the Galileons can play an important role at inflationary or at recent times, in the future, when the universe will asymptotically reach its stable state, they will not have any effect on its evolution.

The plan of the work is the following: In section 2 we briefly review the Galileon cosmological paradigm and in section 3 we perform a detailed phase-space analysis. In section 4 we discuss the cosmological implications and the physical behavior of the scenario. Finally, in section 5 we summarize the obtained results. 


\section{Generalized Galileon cosmology}

In this section we briefly review Galileon cosmology for the most generalized scenario, presenting the background cosmological equations and the conditions for the absence of instabilities [9, 18, 78]. As it is known, in order to avoid the Ostrogradski instability [4] it is desirable to keep the equations of motion at second order in derivatives, and thus the most general 4-dimensional scalar-tensor theories having second-order field equations are described by the Lagrangian [18]

$$
\mathcal{L}=\sum_{i=2}^{5} \mathcal{L}_{i},
$$

where

$$
\begin{aligned}
\mathcal{L}_{2}= & K(\phi, X) \\
\mathcal{L}_{3}= & -G_{3}(\phi, X) \square \phi \\
\mathcal{L}_{4}= & G_{4}(\phi, X) R+G_{4, X}\left[(\square \phi)^{2}-\left(\nabla_{\mu} \nabla_{\nu} \phi\right)\left(\nabla^{\mu} \nabla^{\nu} \phi\right)\right] \\
\mathcal{L}_{5}= & G_{5}(\phi, X) G_{\mu \nu}\left(\nabla^{\mu} \nabla^{\nu} \phi\right) \\
& -\frac{1}{6} G_{5, X}\left[(\square \phi)^{3}-3(\square \phi)\left(\nabla_{\mu} \nabla_{\nu} \phi\right)\left(\nabla^{\mu} \nabla^{\nu} \phi\right)+2\left(\nabla^{\mu} \nabla_{\alpha} \phi\right)\left(\nabla^{\alpha} \nabla_{\beta} \phi\right)\left(\nabla^{\beta} \nabla_{\mu} \phi\right)\right] .
\end{aligned}
$$

The functions $K$ and $G_{i}(i=3,4,5)$ depend on the scalar field $\phi$ and its kinetic energy $X=-\partial^{\mu} \phi \partial_{\mu} \phi / 2$, while $R$ is the Ricci scalar, and $G_{\mu \nu}$ is the Einstein tensor. $G_{i, X}$ and $G_{i, \phi}$ $(i=3,4,5)$ respectively correspond to the partial derivatives of $G_{i}$ with respect to $X$ and $\phi$, namely $G_{i, X} \equiv \partial G_{i} / \partial X$ and $G_{i, \phi} \equiv \partial G_{i} / \partial \phi$. We mention that the above Lagrangian was first discovered by Horndeski [5] in a different but equivalent form [32].

Apart from the above scalar-tensor sectors, in a realistic cosmological scenario one needs to take into account the matter content of the universe, described by the Lagrangian $\mathcal{L}_{m}$, corresponding a perfect fluid with energy density $\rho_{m}$ and pressure $p_{m}$. Then the total action is given by

$$
S=\int d^{4} x \sqrt{-g}\left(\mathcal{L}+\mathcal{L}_{m}\right),
$$

where $g$ is the determinant of the metric $g_{\mu \nu}$.

Let us make here an important comment on the above total action, in which we will focus on the present work. In particular, we do not include a possible coupling between the scalar field and the matter sector. This is usual in many cosmological works [19-22, 4244, 48-50, 67-71]. However, we mention that in its original incarnation the Galileon arises non-minimally coupled to matter $[6,7,11]$. Therefore, strictly speaking, in the present work we view the Galileon theory as a scalar-field, dark-energy, construction, and not as a modified gravity. However, we have in mind that a possible coupling between the Galileon and the matter sector, which allows for the realization of the Vainshtein mechanism, could lead to significantly different cosmological behavior. ${ }^{1}$

\footnotetext{
${ }^{1}$ We thank the referee for this comment.
} 
In the following we impose a flat Friedmann-Robertson-Walker (FRW) background metric of the form $d s^{2}=-N^{2}(t) d t^{2}+a^{2}(t) d \boldsymbol{x}^{2}$, where $t$ is the cosmic time, $x^{i}$ are the comoving spatial coordinates, $N(t)$ is the lapse function, and $a(t)$ is the scale factor. Varying the action (2.6) with respect to $N(t)$ and $a(t)$ respectively, and setting $N=1$, we obtain

$$
\begin{aligned}
& 2 X K_{, X}-K+6 X \dot{\phi} H G_{3, X}-2 X G_{3, \phi}-6 H^{2} G_{4}+24 H^{2} X\left(G_{4, X}+X G_{4, X X}\right) \\
& -12 H X \dot{\phi} G_{4, \phi X}-6 H \dot{\phi} G_{4, \phi}+2 H^{3} X \dot{\phi}\left(5 G_{5, X}+2 X G_{5, X X}\right) \\
& -6 H^{2} X\left(3 G_{5, \phi}+2 X G_{5, \phi X}\right)=-\rho_{m}
\end{aligned}
$$

$$
\begin{aligned}
& K-2 X\left(G_{3, \phi}+\ddot{\phi} G_{3, X}\right)+2\left(3 H^{2}+2 \dot{H}\right) G_{4}-12 H^{2} X G_{4, X}-4 H \dot{X} G_{4, X}-8 \dot{H} X G_{4, X} \\
& -8 H X \dot{X} G_{4, X X}+2(\ddot{\phi}+2 H \dot{\phi}) G_{4, \phi}+4 X G_{4, \phi \phi}+4 X(\ddot{\phi}-2 H \dot{\phi}) G_{4, \phi X} \\
& -2 X\left(2 H^{3} \dot{\phi}+2 H \dot{H} \dot{\phi}+3 H^{2} \ddot{\phi}\right) G_{5, X}-4 H^{2} X^{2} \ddot{\phi} G_{5, X X}+4 H X(\dot{X}-H X) G_{5, \phi X} \\
& +2\left[2(\dot{H} X+H \dot{X})+3 H^{2} X\right] G_{5, \phi}+4 H X \dot{\phi} G_{5, \phi \phi}=-p_{m}
\end{aligned}
$$

where dots denote derivatives with respect to $t$, and we also defined the Hubble parameter $H \equiv \dot{a} / a$. Variation of (2.6) with respect to $\phi(t)$ provides its evolution equation

$$
\frac{1}{a^{3}} \frac{d}{d t}\left(a^{3} J\right)=P_{\phi}
$$

with

$$
\begin{aligned}
J \equiv & \dot{\phi} K_{, X}+6 H X G_{3, X}-2 \dot{\phi} G_{3, \phi}+6 H^{2} \dot{\phi}\left(G_{4, X}+2 X G_{4, X X}\right)-12 H X G_{4, \phi X} \\
& +2 H^{3} X\left(3 G_{5, X}+2 X G_{5, X X}\right)-6 H^{2} \dot{\phi}\left(G_{5, \phi}+X G_{5, \phi X}\right) \\
P_{\phi} \equiv & K_{, \phi}-2 X\left(G_{3, \phi \phi}+\ddot{\phi} G_{3, \phi X}\right)+6\left(2 H^{2}+\dot{H}\right) G_{4, \phi}+6 H(\dot{X}+2 H X) G_{4, \phi X} \\
& -6 H^{2} X G_{5, \phi \phi}+2 H^{3} X \dot{\phi} G_{5, \phi X} .
\end{aligned}
$$

Finally, the evolution equation for matter takes the standard form

$$
\dot{\rho}_{m}+3 H\left(\rho_{m}+p_{m}\right)=0 .
$$

We mention here that the four equations (2.7), (2.8), (2.9) and (2.12), are not independent due to the Bianchi identities. In particular, the scalar equation (2.9) can be acquired from the other three equations [18].

In order to be able to perform the dynamical analysis of Galileon cosmology we need to focus on some more specific models. One class of Galileon scenarios has the above Lagrangian with the ansatzes:

$$
K(\phi, X)=X-V(\phi), G_{3}(\phi, X)=-g(\phi) X, G_{4}(\phi, X)=\frac{1}{2} \frac{1}{8 \pi G}, G_{5}(\phi, X)=0,(2 .
$$

corresponding to the action

$$
S=\int d^{4} x \sqrt{-g}\left[\frac{1}{16 \pi G} R-\frac{1}{2} \partial^{\mu} \phi \partial_{\mu} \phi-V(\phi)-\frac{1}{2} g(\phi) \partial^{\mu} \phi \partial_{\mu} \phi \square \phi+\mathcal{L}_{m}\right] .
$$


Such an action is able to capture the basic, quite general, and more interesting Galileon terms (one could straightforwardly include ansatzes with higher powers of $X$, such as the covariant Galileon model [7], however for simplicity we remain to the above simple but non-trivial Galileon action). In this case, the gravitational field equations (2.7) and (2.8) become

$$
\begin{aligned}
& H^{2}=\frac{8 \pi G}{3}\left(\rho_{D E}+\rho_{m}\right) \\
& \dot{H}=-4 \pi G\left(\rho_{D E}+p_{D E}+\rho_{m}+p_{m}\right),
\end{aligned}
$$

where we have defined the effective dark energy sector with energy density and pressure respectively:

$$
\begin{aligned}
& \rho_{D E}=\frac{\dot{\phi}^{2}}{2}\left(1-6 g H \dot{\phi}+g_{, \phi} \dot{\phi}^{2}\right)+V(\phi), \\
& p_{D E}=\frac{\dot{\phi}^{2}}{2}\left(1+2 g \ddot{\phi}+g_{, \phi} \dot{\phi}^{2}\right)-V(\phi) .
\end{aligned}
$$

The scalar field equation (2.9) becomes

$$
\ddot{\phi}+3 H \dot{\phi}+2 g_{, \phi} \dot{\phi}^{2} \ddot{\phi}+\frac{1}{2} g_{, \phi \phi} \dot{\phi}^{4}-3 g \dot{H} \dot{\phi}^{2}-6 g H \dot{\phi} \ddot{\phi}-9 g H^{2} \dot{\phi}^{2}+V_{, \phi}=0
$$

and we can immediately see that using $(2.17),(2.18)$ it can be rewritten to the standard form

$$
\dot{\rho}_{D E}+3 H\left(\rho_{D E}+p_{D E}\right)=0 .
$$

Furthermore, we can define the dark energy equation-of-state parameter as

$$
w_{D E} \equiv \frac{p_{D E}}{\rho_{D E}}=\frac{\frac{\dot{\phi}^{2}}{2}\left(1+2 g \ddot{\phi}+g_{, \phi} \dot{\phi}^{2}\right)-V(\phi)}{\frac{\dot{\phi}^{2}}{2}\left(1-6 g H \dot{\phi}+g_{, \phi} \dot{\phi}^{2}\right)+V(\phi)} .
$$

One can clearly see that in this scenario, according to the form of $g(\phi), w_{D E}$ can be quintessence-like, phantom-like, or experience the phantom divide crossing during the evolution, which is a great advantage of Galileon cosmology.

Without loss of generality in the following we restrict the analysis to the dust matter case, that is we assume that $w_{m} \equiv p_{m} / \rho_{m}=0$. In this case it is convenient to introduce two additional quantities with great physical significance, namely the "total" equation-of-state parameter

$$
w_{t o t} \equiv \frac{p_{D E}}{\rho_{D E}+\rho_{m}}=\frac{8 \pi G}{3 H^{2}}\left[\frac{\dot{\phi}^{2}}{2}\left(1+2 g \ddot{\phi}+g_{, \phi} \dot{\phi}^{2}\right)-V(\phi)\right],
$$

and the deceleration parameter

$$
q \equiv-1-\frac{\dot{H}}{H^{2}}=\frac{1}{2}+\frac{3}{2} w_{t o t}
$$

We close this section by mentioning that in order for the above scenario to be free of ghosts and Laplacian instabilities, and thus cosmologically viable, two conditions must be 
satisfied $[18,20,62]$. In particular, using the ansatzes (2.13) and units where $\kappa \equiv 8 \pi G=1$, these write as [18]

$$
c_{S}^{2} \equiv \frac{6 w_{1} H-3 w_{1}^{2}-6 \dot{w}_{1}-6 \rho_{m}}{4 w_{2}+9 w_{1}^{2}} \geq 0
$$

for the avoidance of Laplacian instabilities associated with the scalar field propagation speed, and

$$
Q_{S} \equiv \frac{\left(4 w_{2}+9 w_{1}^{2}\right)}{3 w_{1}^{2}}>0
$$

for the absence of ghosts, where in our case

$$
\begin{aligned}
w_{1} & \equiv g \dot{\phi}^{3}+2 H \\
w_{2} & \equiv 3 \dot{\phi}^{2}\left[\frac{1}{2}+g_{, \phi} \dot{\phi}^{2}-6 H g \dot{\phi}\right]-9 H^{2} .
\end{aligned}
$$

Finally, we stress that according to $(2.21)$ and $(2.24),(2.25)$ the phantom phase can be free of instabilities and thus cosmologically viable, as it was already shown for Galileon cosmology [18].

\section{Phase space analysis}

In this section we perform a detailed phase-space and stability analysis of generalized Galileon cosmology. As usual, we first transform the above dynamical system into its autonomous form $\mathbf{X}^{\prime}=\mathbf{f}(\mathbf{X})$ [107-111], where $\mathbf{X}$ is the column vector constituted by suitably chosen auxiliary variables, $\mathbf{f}(\mathbf{X})$ the corresponding column vector of the autonomous equations, with primes corresponding to derivatives with respect to $M=\ln a$. Next we extract its critical points $\mathbf{X}_{\mathbf{c}}$ demanding $\left.\mathbf{X}^{\prime}\right|_{\mathbf{X}=\mathbf{X}_{\mathbf{c}}}=\mathbf{0}$, and in order to determine their stability properties we expand around $\mathbf{X}_{\mathbf{c}}$ as $\mathbf{X}=\mathbf{X}_{\mathbf{c}}+\mathbf{U}$, with $\mathbf{U}$ the column vector of the perturbations. Therefore, for each critical point we expand the perturbation equations up to first order as $\mathbf{U}^{\prime}=\mathbf{Q} \cdot \mathbf{U}$, where the matrix $\mathbf{Q}$ contains the coefficients of the perturbation equations. Lastly, the eigenvalues of $\mathbf{Q}$ calculated at each critical point determine its type and stability.

In the scenario at hand we introduce the auxiliary variables:

$$
\begin{aligned}
& x=\frac{\kappa \dot{\phi}}{\sqrt{6} H} \\
& y=\frac{\kappa \sqrt{V(\phi)}}{\sqrt{3} H} \\
& z=g(\phi) H \dot{\phi} \\
& v=\frac{1}{\phi} .
\end{aligned}
$$

Using these variables the Friedmann equation (2.15) becomes

$$
(1-6 z) x^{2}+y^{2}+\frac{\sqrt{6} z g^{\prime}(\phi) x^{3}}{g(\phi)}+\frac{\rho_{m}}{3 H^{2}}=1 .
$$


Moreover, using (3.2) and (2.17) we can write the matter and dark energy density parameters as:

$$
\begin{aligned}
& \Omega_{m} \equiv \frac{\rho_{m}}{3 H^{2}}=1-\left[(1-6 z) x^{2}+y^{2}+\frac{\sqrt{6} z g^{\prime}(\phi) x^{3}}{g(\phi)}\right] \\
& \Omega_{D E} \equiv \frac{\kappa^{2} \rho_{D E}}{3 H^{2}}=(1-6 z) x^{2}+y^{2}+\frac{\sqrt{6} z g^{\prime}(\phi) x^{3}}{g(\phi)} .
\end{aligned}
$$

Note that in the limit $g(\phi) \rightarrow 0$ the above quantities are well-defined, and they coincide with the usual quintessence ones [107].

In order to proceed forward we need to consider a specific scalar-field potential $V(\phi)$ and a specific coupling function $g(\phi)$ with the Galileon term. Concerning $V(\phi)$ the usual assumption in dynamical investigations in the literature is to assume an exponential potential of the form

$$
V(\phi)=V_{0} e^{\lambda_{V} \phi},
$$

since exponential potentials are known to be significant in various cosmological models [107-113] (equivalently, but more generally, we could use potentials satisfying $\lambda_{V}=$ $-\frac{1}{V(\phi)} \frac{\partial V(\phi)}{\partial \phi} \approx$ const, which is the case for arbitrary but nearly flat potentials [114-116]). Concerning $g(\phi)$, and in order to remain general, we will consider two ansatzes, namely the exponential one

$$
g(\phi)=g_{0} e^{\lambda_{g} \phi}
$$

and the power-law one

$$
g(\phi)=g_{0} \phi^{n} .
$$

The corresponding analysis will be performed separately in the following two subsections.

\subsection{Scenario 1: Exponential potential and exponential coupling function}

In this subsection we consider the exponential potential (3.4) and the exponential coupling function (3.5). In this case, using the auxiliary variables (3.1), the equations (2.15), (2.16) and (2.19) can be transformed to the autonomous form

$$
\begin{aligned}
x^{\prime}=\left[18 z^{2} x^{3}+\right. & \left.4 \sqrt{6} z \lambda_{g} x^{2}+(2-12 z) x\right]^{-1}\left\{18 z^{2} \lambda_{g}^{2} x^{6}+\left(9 \sqrt{6} z \lambda_{g}-27 \sqrt{6} z^{2} \lambda_{g}\right) x^{5}\right. \\
& +\left[54 z^{2}+\left(-6 \lambda_{g}^{2}-45\right) z+3\right] x^{4}+\left[6 \sqrt{6} z \lambda_{g}-3 \sqrt{6} y^{2} z\left(2 \lambda_{g}+\lambda_{V}\right)\right] x^{3} \\
& \left.+\left[(27 z-3) y^{2}-9 z-3\right] x^{2}-\sqrt{6} y^{2} \lambda_{V} x\right\} \\
y^{\prime}=\left[18 z^{2} x^{3}+4\right. & \left.\sqrt{6} z \lambda_{g} x^{2}+(2-12 z) x\right]^{-1}\left\{18 y z^{2} \lambda_{g}^{2} x^{5}+y x^{4}\left[9 \sqrt{6}\left(\lambda_{V}-2 \lambda_{g}\right) z^{2}+9 \sqrt{6} \lambda_{g} z\right]\right. \\
& +y x^{3}\left[54 z^{2}+12\left(\lambda_{g} \lambda_{V}-3\right) z+3\right]+x\left[(18 z-3) y^{3}+(3-18 z) y\right] \\
& \left.+x^{2}\left\{y\left[6 \sqrt{6} z\left(\lambda_{g}-\lambda_{V}\right)+\sqrt{6} \lambda_{V}\right]-3 \sqrt{6} y^{3} z\left(2 \lambda_{g}+\lambda_{V}\right)\right\}\right\}
\end{aligned}
$$




$$
\begin{aligned}
z^{\prime}=\left[18 z^{2} x^{3}\right. & \left.+4 \sqrt{6} z \lambda_{g} x^{2}+(2-12 z) x\right]^{-1}\left\{-18 z^{3} \lambda_{g}^{2} x^{5}+\left(27 \sqrt{6} z^{3} \lambda_{g}-9 \sqrt{6} z^{2} \lambda_{g}\right) x^{4}\right. \\
& +x^{3}\left[-54 z^{3}+9\left(2 \lambda_{g}^{2}+3\right) z^{2}-3 z\right]+x\left[\left(3 z-9 z^{2}\right) y^{2}+27 z^{2}-9 z\right] \\
& \left.+x^{2}\left[-18 \sqrt{6} \lambda_{g} z^{2}+3 \sqrt{6} y^{2}\left(2 \lambda_{g}+\lambda_{V}\right) z^{2}+2 \sqrt{6} \lambda_{g} z\right]-\sqrt{6} y^{2} z \lambda_{V}\right\}
\end{aligned}
$$

defined in the (non-compact) phase space $\Psi=\left\{(x, y, z): y>0, z\left(\sqrt{6} x \lambda_{g}-6\right) x^{2}+x^{2}+y^{2} \leq 1\right\}$. Note that in this case the variable $v$ is not needed.

Using (2.17) and the Friedmann equation (3.2), we can write the density parameters as:

$$
\begin{aligned}
& \Omega_{m} \equiv \frac{\rho_{m}}{3 H^{2}}=1-\left[(1-6 z) x^{2}+y^{2}+\sqrt{6} z x^{3} \lambda_{g}\right] \\
& \Omega_{D E} \equiv \frac{\kappa^{2} \rho_{D E}}{3 H^{2}}=(1-6 z) x^{2}+y^{2}+\sqrt{6} z x^{3} \lambda_{g}
\end{aligned}
$$

while for the dark-energy equation-of-state parameter (2.21) we obtain:

$$
w_{D E}=\frac{6 z^{2} \lambda_{g}^{2} x^{4}+3 \sqrt{6}(1-2 z) z \lambda_{g} x^{3}+[3 z(3 z-4)+1] x^{2}-\sqrt{6} y^{2} z\left(2 \lambda_{g}+\lambda_{V}\right) x+y^{2}(6 z-1)}{\left[z x^{2}\left(\sqrt{6} x \lambda_{g}-6\right)+x^{2}+y^{2}\right]\left[z\left(9 z x^{2}+2 \sqrt{6} \lambda_{g} x-6\right)+1\right]} .
$$

As we mentioned above, according to the variable $z$, that is according to the coupling function $g(\phi)$, in this scenario $w_{D E}$ can be quintessence-like, phantom-like, or experience the phantom divide crossing during the evolution. Furthermore, the total equation-of-state parameter (2.22) becomes

$$
\begin{aligned}
& w_{t o t} \equiv \frac{p_{D E}}{\rho_{D E}+\rho_{m}}=w_{D E} \Omega_{D E}= \\
& =\frac{6 z^{2} \lambda_{g}^{2} x^{4}+3 \sqrt{6}(1-2 z) z \lambda_{g} x^{3}+[3 z(3 z-4)+1] x^{2}-\sqrt{6} y^{2} z\left(2 \lambda_{g}+\lambda_{V}\right) x+y^{2}(6 z-1)}{z\left(9 z x^{2}+2 \sqrt{6} \lambda_{g} x-6\right)+1},
\end{aligned}
$$

and the deceleration parameter (2.23) reads

$$
\begin{aligned}
q \equiv-1-\frac{\dot{H}}{H^{2}}= & \frac{1}{2}+\frac{3}{2} w_{t o t} \\
= & \left\{2 z\left(9 z x^{2}+2 \sqrt{6} \lambda_{g} x-6\right)+2\right\}^{-1}\left\{9 \sqrt{6}(1-2 z) z \lambda_{g} x^{3}+[36(z-1) z+3] x^{2}\right. \\
& \left.\quad+18 z^{2} \lambda_{g}^{2} x^{4}+\sqrt{6} z\left[\left(2-6 y^{2}\right) \lambda_{g}-3 y^{2} \lambda_{V}\right] x+\left(3 y^{2}-1\right)(6 z-1)\right\} .
\end{aligned}
$$

Finally, the two instability-related quantities (2.24) and (2.25) are respectively written as

$$
\begin{aligned}
c_{S}^{2}= & \left\{x\left[z\left(9 z x^{2}+2 \sqrt{6} \lambda_{g} x-6\right)+1\right]^{2}\right\}^{-1}\left\{3 \sqrt{6} z^{3} \lambda_{g} x^{4}+3 z^{2} x^{3}\left(2 \lambda_{g}^{2}-6 z+5\right)\right. \\
& \left.-27 z^{4} x^{5}+2 \sqrt{6}(1-4 z) z \lambda_{g} x^{2}+\left\{z\left[3 z\left(5-3 y^{2}\right)-4\right]+1\right\} x+\sqrt{6} y^{2} z \lambda_{V}\right\}(3.1
\end{aligned}
$$

and

$$
Q_{S}=\frac{3 x^{2}\left[z\left(9 z x^{2}+2 \sqrt{6} \lambda_{g} x-6\right)+1\right]}{\left(3 z x^{2}+1\right)^{2}}
$$




\begin{tabular}{|c|c|c|c|c|c|}
\hline Cr. P. & $x_{c}$ & $y_{c}$ & $z_{c}$ & Exist for & Stability \\
\hline \hline$A^{+}$ & +1 & 0 & 0 & always & $\begin{array}{c}\text { unstable for } \lambda_{V}>-\sqrt{6}, \lambda_{g}>\sqrt{6} \\
\text { saddle point otherwise }\end{array}$ \\
\hline$A^{-}$ & -1 & 0 & 0 & always & $\begin{array}{c}\text { unstable for } \lambda_{V}<\sqrt{6}, \lambda_{g}<-\sqrt{6} \\
\text { saddle point otherwise }\end{array}$ \\
\hline$B^{+}$ & $\frac{\lambda_{g}+\sqrt{\lambda^{2}-6}}{\sqrt{6}}$ & 0 & $\frac{3-\alpha^{-}\left(\lambda_{g}\right)}{9}$ & $\lambda_{g}^{2} \geq 6$ & $\begin{array}{c}\text { unstable for } \lambda_{g}>\sqrt{6}, \lambda_{V}>-\lambda_{g} \\
\text { saddle point otherwise }\end{array}$ \\
\hline$B^{-}$ & $\frac{\lambda_{g}-\sqrt{\lambda_{g}{ }^{2}-6}}{\sqrt{6}}$ & 0 & $\frac{3-\alpha^{+}\left(\lambda_{g}\right)}{9}$ & $\lambda_{g}^{2} \geq 6$ & $\begin{array}{c}\text { unstable for } \lambda_{g}<-\sqrt{6}, \lambda_{V}<-\lambda_{g} \\
\text { saddle point otherwise }\end{array}$ \\
\hline$C$ & $-\frac{\lambda_{V}}{\sqrt{6}}$ & $\sqrt{1-\frac{\lambda_{V}{ }^{2}}{6}}$ & 0 & $0<\lambda_{V}^{2} \leq 6$ & $\begin{array}{c}\text { stable node for }-\sqrt{3}<\lambda_{V}<0, \lambda_{g}<-\lambda_{V} \\
\text { stable node for } 0<\lambda_{V}<\sqrt{3}, \lambda_{g}>-\lambda_{V} \\
\text { saddle point otherwise }\end{array}$ \\
\hline$C_{0}$ & 0 & 1 & 0 & $\lambda_{V}=0$ & stable node \\
\hline$D$ & $-\frac{\sqrt{6}}{2 \lambda_{V}}$ & $\frac{\sqrt{6}}{2 \lambda_{V}}$ & 0 & $\lambda_{V}^{2} \geq 3$ & $\begin{array}{c}\text { stable node for }-\sqrt{\frac{24}{7}} \leq \lambda_{V}<-\sqrt{3}, \lambda_{g}<-\lambda_{V} \\
\text { stable node for } \sqrt{3}<\lambda_{V}<\sqrt{\frac{24}{7}}, \lambda_{g}>-\lambda_{V} \\
\text { stable spiral for } \lambda_{V}<-\sqrt{\frac{24}{7}}, \lambda_{g}<-\lambda_{V} \\
\text { stable spiral for } \lambda_{V}>\sqrt{\frac{24}{7}}, \lambda_{g}>-\lambda_{V}\end{array}$ \\
\hline$O_{1}$ & 0 & 0 & 0 & always & saddle \\
\hline
\end{tabular}

Table 1. Scenario 1: Exponential potential and exponential coupling function. The real and physically meaningful critical points of the autonomous system (3.7)-(3.9), and their existence and stability conditions. We have introduced the functions $\alpha^{ \pm}\left(\lambda_{g}\right)=\lambda_{g}^{2} \pm \lambda_{g} \sqrt{\lambda_{g}^{2}-6}$.

\subsubsection{Finite phase-space analysis}

Let us now proceed to the phase-space analysis. The real and physically meaningful critical points $\left(x_{c}, y_{c}, z_{c}\right)$ of the autonomous system (3.7)-(3.9) (that is corresponding to an expanding universe, and thus possessing $H>0$, with $\left.0 \leq \Omega_{D E} \leq 1\right)$, are obtained by setting the left hand sides of the equations to zero, and they are presented in Table 1. In the same table we provide their existence conditions. For each critical point of Table 1 we calculate the $3 \times 3$ matrix $\mathbf{Q}$ of the linearized perturbation equations of the system (3.7)-(3.9), and in order to determine the type and stability of the point we examine the sign of the real part of the eigenvalues of $\mathbf{Q}$. The details of the analysis and the various eigenvalues are presented in Appendix A.1, and in Table 1 we summarize the stability results. Moreover, for each critical point we calculate the values of $\Omega_{D E}, w_{D E}, w_{t o t}$ and $q$ given by (3.10)-(3.13), as well as the instability-related quantities $c_{S}^{2}$ and $Q_{S}$ given in (3.14),(3.15), and we summarize the results in Table 2.

\subsubsection{Phase-space analysis at infinity}

Since the dynamical system (3.7)-(3.9) is non-compact, there could be features in the asymptotic regime which are non-trivial for the global dynamics. Therefore, in order to 


\begin{tabular}{|c|c|c|c|c|c|c|}
\hline Cr. P. & $\Omega_{D E}$ & $w_{D E}$ & $w_{t o t}$ & $\mathrm{q}$ & $c_{S}^{2}$ & $Q_{S}$ \\
\hline \hline$A^{ \pm}$ & 1 & 1 & 1 & 2 & 1 & 3 \\
\hline$B^{ \pm}$ & 1 & $-1+\frac{\alpha^{ \pm}\left(\lambda_{g}\right)}{3}$ & $-1+\frac{\alpha^{ \pm}\left(\lambda_{g}\right)}{3}$ & $-1+\frac{\alpha^{ \pm}\left(\lambda_{g}\right)}{2}$ & 0 & arbitrary \\
\hline$C$ & 1 & $-1+\frac{\lambda_{V}^{2}}{3}$ & $-1+\frac{\lambda_{V}^{2}}{3}$ & $-1+\frac{\lambda_{V}^{2}}{2}$ & 1 & $\frac{\lambda_{V}^{2}}{2}$ \\
\hline$C_{0}$ & 1 & -1 & -1 & -1 & 1 & 0 \\
\hline$D$ & $\frac{3}{\lambda_{V}^{2}}$ & 0 & 0 & $\frac{1}{2}$ & 1 & $\frac{9}{2 \lambda_{V}^{2}}$ \\
\hline $\mathrm{fz} O_{1}$ & 0 & arbitrary & 0 & $\frac{1}{2}$ & 1 & 0 \\
\hline
\end{tabular}

Table 2. Scenario 1: Exponential potential and exponential coupling function. The real and physically meaningful critical points of the autonomous system (3.7)-(3.9), and the corresponding values of the dark-energy density parameter $\Omega_{D E}$, of the dark-energy equation-of-state parameter $w_{D E}$, of the total equation-of-state parameter $w_{t o t}$ and of the deceleration parameter $q$. The last two columns contain the instability-related parameters $c_{S}^{2}$ and $Q_{S}$ (from (3.14),(3.15)), which must respectively be non-negative and positive for a scenario free of ghosts and instabilities. We have introduced the functions $\alpha^{ \pm}\left(\lambda_{g}\right)=\lambda_{g}^{2} \pm \lambda_{g} \sqrt{\lambda_{g}^{2}-6}>0$.

complete the phase-space analysis we have to extend the investigation using the Poincaré central projection method [117].

We consider the Poincaré variables

$$
x_{r}=\rho \cos \theta \sin \psi, z_{r}=\rho \sin \theta \sin \psi, y_{r}=\rho \cos \psi,
$$

with $\rho=\frac{r}{\sqrt{1+r^{2}}}, r=\sqrt{x^{2}+y^{2}+z^{2}}, \theta \in[0,2 \pi]$, and $-\frac{\pi}{2} \leq \psi \leq \frac{\pi}{2}$ (we restrict the $\psi$ angle to this range since the physical region is given by $y>0$ ) [117-120]. Therefore, the points at "infinity" $(r \rightarrow+\infty)$ are those with $\rho \rightarrow 1$. Moreover, the physical phase space is now given by $\left(x_{r}, y_{r}, z_{r}\right) \in[-1,1] \times[0,1] \times[-1,1]$, such that

$$
\frac{x_{r}^{2}+y_{r}^{2}}{1-x_{r}^{2}-y_{r}^{2}-z_{r}^{2}}-\frac{x_{r}^{2} z_{r}\left(\sqrt{6} \lambda_{g} x_{r}-6 x_{r}^{2}-6 y_{r}^{2}-6 z_{r}^{2}+6\right)}{\left(1-x_{r}^{2}-y_{r}^{2}-z_{r}^{2}\right)^{5 / 2}} \leq 1,
$$

and $x_{r}^{2}+y_{r}^{2}+z_{r}^{2} \leq 1$.

Inverting relations (3.16) and substituting into (3.10),(3.11), we obtain the dark energy density and equation-of-state parameters as a function of the Poincaré variables, namely:

$$
\begin{gathered}
\Omega_{D E}=\frac{x_{r}^{2}+y_{r}^{2}}{1-x_{r}^{2}-y_{r}^{2}-z_{r}^{2}}-\frac{x_{r}^{2} z_{r}\left(\sqrt{6} \lambda_{g} x_{r}-6 x_{r}^{2}-6 y_{r}^{2}-6 z_{r}^{2}+6\right)}{\left(1-x_{r}^{2}-y_{r}^{2}-z_{r}^{2}\right)^{5 / 2}}, \\
w_{D E}=\left\{\left[\sqrt{6} z_{r} \lambda_{g} x_{r}^{3}+\left(\zeta-6 z_{r} \sqrt{\zeta}\right) x_{r}^{2}+y_{r}^{2} \zeta\right]\left(9 x_{r}^{2} z_{r}^{2}-6 \zeta^{3 / 2} z_{r}+2 \sqrt{6} x_{r} \zeta \lambda_{g} z_{r}+\zeta^{2}\right)\right\}^{-1} \\
\zeta\left\{6 z_{r}^{2} \lambda_{g}^{2} x_{r}^{4}+3 \sqrt{6} z_{r}\left(\sqrt{\zeta}-2 z_{r}\right) \sqrt{\zeta} \lambda_{g} x_{r}^{3}+\zeta\left(9 z_{r}^{2}-12 \sqrt{\zeta} z_{r}+\zeta\right) x_{r}^{2}\right. \\
\left.-\sqrt{6} y_{r}^{2} z_{r} \zeta\left(2 \lambda_{g}+\lambda_{V}\right) x_{r}+y_{r}^{2}\left(6 z_{r}-\sqrt{\zeta}\right) \zeta^{3 / 2}\right\},(3.19)
\end{gathered}
$$




\begin{tabular}{|c|c|c|c|c|c|c|c|c|c|c|}
\hline Cr. P. & $x_{r}$ & $y_{r}$ & $z_{r}$ & Stability & $\Omega_{D E}$ & $w_{D E}$ & $w_{t o t}$ & $\mathrm{q}$ & $c_{S}^{2}$ & $Q_{S}$ \\
\hline \hline$K^{ \pm}$ & 0 & 0 & \pm 1 & unstable & 0 & arbitrary & 0 & $\frac{1}{2}$ & arbitrary & 0 \\
\hline
\end{tabular}

Table 3. Scenario 1: Exponential potential and exponential coupling function. The real and physically meaningful critical points at infinity of the autonomous system (3.7)-(3.9), and the corresponding values of the dark-energy density parameter $\Omega_{D E}$, of the dark-energy equation-of-state parameter $w_{D E}$, of the total equation-of-state parameter $w_{t o t}$ and of the deceleration parameter $q$. The last two columns contain the instability-related parameters $c_{S}^{2}$ and $Q_{S}$ (from (3.22),(3.23)), which must respectively be non-negative and positive for a scenario free of ghosts and instabilities.

with $\zeta=1-x_{r}^{2}-y_{r}^{2}-z_{r}^{2}$, and similarly substituting into (3.12), (3.13) we obtain the corresponding expressions for the total equation-of-state and deceleration parameters:

$$
\begin{array}{r}
w_{\text {tot }}=\left[\zeta\left(9 x_{r}^{2} z_{r}^{2}-6 \zeta^{3 / 2} z_{r}+2 \sqrt{6} x_{r} \zeta \lambda_{g} z_{r}+\zeta^{2}\right)\right]^{-1}\left\{3 \sqrt{6} z_{r}\left(\sqrt{\zeta}-2 z_{r}\right) \sqrt{\zeta} \lambda_{g} x_{r}^{3}\right. \\
+\zeta\left(9 z_{r}^{2}-12 \sqrt{\zeta} z_{r}+\zeta\right) x_{r}^{2}-\sqrt{6} y_{r}^{2} z_{r} \zeta\left(2 \lambda_{g}+\lambda_{V}\right) x_{r} \\
\left.+6 z_{r}^{2} \lambda_{g}^{2} x_{r}^{4}+y_{r}^{2}\left(6 z_{r}-\sqrt{\zeta}\right) \zeta^{3 / 2}\right\} \\
q=\left[2 \zeta\left(9 x_{r}^{2} z_{r}^{2}-6 \zeta^{3 / 2} z_{r}+2 \sqrt{6} x_{r} \zeta \lambda_{g} z_{r}+\zeta^{2}\right)\right]^{-1}\left\{9 \sqrt{6} z_{r}\left(\sqrt{\zeta}-2 z_{r}\right) \sqrt{\zeta} \lambda_{g} x_{r}^{3}\right. \\
+3 \zeta\left(12 z_{r}^{2}-12 \sqrt{\zeta} z_{r}+\zeta\right) x_{r}^{2}+\sqrt{6} z_{r} \zeta\left[2 \zeta \lambda_{g}-3 y_{r}^{2}\left(2 \lambda_{g}+\lambda_{V}\right)\right] x_{r} \\
\left.+18 z_{r}^{2} \lambda_{g}^{2} x_{r}^{4}+\zeta^{3 / 2}\left(\sqrt{\zeta}-6 z_{r}\right)\left(\zeta-3 y_{r}^{2}\right)\right\}
\end{array}
$$

Finally, substitution into (3.14),(3.15) provides the corresponding expressions for the instabilityrelated quantities:

$$
\begin{gathered}
c_{S}^{2}=\left[x_{r}\left(9 x_{r}^{2} z_{r}^{2}-6 \zeta^{3 / 2} z_{r}+2 \sqrt{6} x_{r} \zeta \lambda_{g} z_{r}+\zeta^{2}\right)^{2}\right]^{-1}\left\{-27 z_{r}^{4} x_{r}^{5}+3 \sqrt{6} z_{r}^{3} \zeta \lambda_{g} x_{r}^{4}\right. \\
+3 z_{r}^{2} \zeta^{3 / 2}\left[\sqrt{\zeta}\left(2 \lambda_{g}^{2}+5\right)-6 z_{r}\right] x_{r}^{3}+2 \sqrt{6} z_{r}\left(\sqrt{\zeta}-4 z_{r}\right) \zeta^{5 / 2} \lambda_{g} x_{r}^{2} \\
\left.+\zeta^{2}\left[\zeta\left(15 z_{r}^{2}-4 \sqrt{\zeta} z_{r}+\zeta\right)-9 y_{r}^{2} z_{r}^{2}\right] x_{r}+\sqrt{6} y_{r}^{2} z_{r} \zeta^{3} \lambda_{V}\right\} \\
Q_{S}=\frac{3 x_{r}^{2}\left(9 x_{r}^{2} z_{r}^{2}-6 \zeta^{3 / 2} z_{r}+2 \sqrt{6} x_{r} \zeta \lambda_{g} z_{r}+\zeta^{2}\right)}{\left(3 z_{r} x_{r}^{2}+\zeta^{3 / 2}\right)^{2}}
\end{gathered}
$$

Therefore, performing the analysis described in Appendix A.2 we conclude that there are two physical critical points at infinity, namely $K^{ \pm}$. These critical points, along with their stability conditions and the corresponding values of observables, are presented in Table 3.

\subsection{Scenario 2: Exponential potential and power-law coupling function}

Let us consider an exponential potential $V(\phi)=V_{0} e^{\lambda_{V} \phi}$ and a power-law coupling function $g(\phi)=g_{0} \phi^{n}$. In this case, using the auxiliary variables (3.1), the equations (2.15), (2.16) 
and (2.19) can be transformed to the autonomous form:

$$
\begin{aligned}
x^{\prime}=[2 \sqrt{6} & \left.+24 x v z n-12 z \sqrt{6}+18 \sqrt{6} x^{2} z^{2}\right]^{-1}\left\{18 \sqrt{6} n(n+1) v^{2} z^{2} x^{5}-6 y^{2} \lambda_{V}\right. \\
+ & 54 v z n x^{4}(1-3 z)+3 \sqrt{6} x^{3}\left\{18 z^{2}+\left[2 n(1-n) v^{2}-15\right] z+1\right\} \\
- & \left.18 z x^{2}\left[\left(2 v n+\lambda_{V}\right) y^{2}-2 v n\right]+3 \sqrt{6} x\left[(9 z-1) y^{2}-3 z-1\right]\right\} \\
y^{\prime}=\left[\begin{array}{l}
2 \sqrt{6} \\
+
\end{array}\right. & \left.24 x v z n-12 z \sqrt{6}+18 \sqrt{6} x^{2} z^{2}\right]^{-1}\left\{18 \sqrt{6} n(n+1) v^{2} z^{2} y x^{4}\right. \\
+ & 3 \sqrt{6} y x^{2}\left[18 z^{2}+4\left(\lambda_{V} n v-3\right) z+1\right]+3 \sqrt{6}(6 z-1) y^{3} \\
& -6 x\left\{3 z y^{3}\left(2 n v+\lambda_{V}\right)-y\left[6 z\left(n v-\lambda_{V}\right)+\lambda_{V}\right]\right\} \\
& \left.+3 \sqrt{6} y(1-6 z)+54 y x^{3}\left[\left(\lambda_{V}-2 n v\right) z^{2}+v z n\right]\right\} \\
z^{\prime}=[2 x & \left.\left(\sqrt{6}+12 x v z n-6 z \sqrt{6}+9 \sqrt{6} x^{2} z^{2}\right)\right]^{-1}\left\{-18 \sqrt{6} n(n+1) v^{2} z^{3} x^{5}\right. \\
+ & 54 n v z^{2} x^{4}(3 z-1)-3 \sqrt{6} z x^{3}\left\{18 z^{2}-\left[2 n(1+3 n) v^{2}+9\right] z+1\right\} \\
+ & 6 z x^{2}\left[\left(6 n v+3 \lambda_{V}\right) z y^{2}-18 n v z+2 v n\right] \\
+ & \left.3 z \sqrt{6} x\left[y^{2}(1-3 z)+9 z-3\right]-6 z y^{2} \lambda_{V}\right\} \\
v^{\prime}=- & \sqrt{6} v^{2} x,
\end{aligned}
$$

defined in the non-compact phase space

$$
\Psi=\left\{(x, y, z, v): y>0, z(\sqrt{6} n v x-6) x^{2}+x^{2}+y^{2} \leq 1, v \in \mathbb{R}\right\} .
$$

Note that contrary to the previous Scenario 1, in the case at hand we do need the fourth auxiliary variable $v$.

Using (3.2) and (2.17) we can write the density parameters as:

$$
\begin{aligned}
& \Omega_{m} \equiv \frac{\rho_{m}}{3 H^{2}}=1-\left[(1-6 z) x^{2}+y^{2}+\sqrt{6} n v z x^{3}\right] \\
& \Omega_{D E} \equiv \frac{\kappa^{2} \rho_{\phi}}{3 H^{2}}=(1-6 z) x^{2}+y^{2}+\sqrt{6} n v z x^{3},
\end{aligned}
$$

while for the dark-energy equation-of-state parameter (2.21) we obtain:

$$
\begin{gathered}
w_{D E}=\left\{\left(\sqrt{6} x^{2}-6 \sqrt{6} z x^{2}+6 v x^{3} z n+\sqrt{6} y^{2}\right)\left(\sqrt{6}+12 x v z n-6 z \sqrt{6}+9 \sqrt{6} x^{2} z^{2}\right)\right\}^{-1} \\
6\left[6(1+n) n v^{2} z^{2} x^{4}+3 \sqrt{6} n(1-2 z) v x^{3} z+\left(1+9 z^{2}-12 z\right) x^{2}\right. \\
\left.-\sqrt{6}\left(2 n v+6 \lambda_{V}\right) z y^{2} x+(6 z-1) y^{2}\right] .
\end{gathered}
$$

Furthermore, the total equation-of-state parameter (2.22) parameter reads

$$
\begin{array}{r}
w_{t o t}=\left(1+2 \sqrt{6} x v z n-6 z+9 x^{2} z^{2}\right)^{-1}\left[6(1+n) n v^{2} z^{2} x^{4}+3 \sqrt{6} n(1-2 z) v x^{3} z\right. \\
\left.+\left(1+9 z^{2}-12 z\right) x^{2}-\sqrt{6}\left(2 n v+6 \lambda_{V}\right) z y^{2} x+(6 z-1) y^{2}\right],
\end{array}
$$


and the deceleration parameter (2.23) becomes

$$
q=\frac{1}{2}+\frac{3}{2} w_{t o t}
$$

Finally, from $(2.24),(2.25)$ we find

$$
\begin{array}{r}
c_{S}^{2}=\left[x\left(1+2 \sqrt{6} z x n v-6 z+9 x^{2} z^{2}\right)^{2}\right]^{-1}\left\{-27 x^{5} z^{4}+3 \sqrt{6} x^{4} z^{3} n v\right. \\
+3 z^{2} x^{3}\left[-6 z+5+2 n(n-1) v^{2}\right]+2 \sqrt{6} z n v(1-4 z) x^{2} \\
\left.+x\left(15 z^{2}-9 y^{2} z^{2}-4 z+1\right)+z \sqrt{6} y^{2} \lambda_{V}\right\}
\end{array}
$$

and

$$
Q_{S}=\frac{\left(3+6 \sqrt{6} z x n v-18 z+27 x^{2} z^{2}\right) x^{2}}{\left(3 x^{2} z+1\right)^{2}}
$$

\subsubsection{Finite phase-space analysis}

Let us now proceed to the phase-space analysis. The real and physically meaningful critical points $\left(x_{c}, y_{c}, z_{c}, v_{c}\right)$ of the autonomous system (3.24)-(3.27) (that is corresponding to an expanding universe, and thus possessing $H>0$, with $\left.0 \leq \Omega_{D E} \leq 1\right)$, are presented in Table 4, along with their existence conditions. The details of the analysis and the various eigenvalues are presented in Appendix B.1. Furthermore, in Table 5 we display the corresponding values of the observables $\Omega_{D E}, w_{D E}, w_{t o t}$ and $q$ given by (3.28)-(3.31), as well as the instability-related quantities $c_{S}^{2}$ and $Q_{S}$ given in (3.32),(3.33). We mention

\begin{tabular}{|c|c|c|c|c|c|c|}
\hline Cr. P. & $x_{c}$ & $y_{c}$ & $z_{c}$ & $v_{c}$ & Exist for & Stability \\
\hline \hline$E^{ \pm}$ & \pm 1 & 0 & 0 & 0 & always & saddle point \\
\hline$F$ & $-\frac{\lambda_{V}}{6}$ & $\sqrt{1-\frac{\lambda_{V}^{2}}{6}}$ & 0 & 0 & $0<\lambda_{V}^{2} \leq 6$ & $\begin{array}{c}\text { stable node for } \lambda_{V}^{2}<3 \\
\text { saddle point for } 3<\lambda_{V}^{2}<6\end{array}$ \\
\hline$F_{0}$ & 0 & 1 & 0 & 0 & $\lambda_{V}=0$ & $\begin{array}{c}\text { stable (not asymptotically for } n \neq 0 \text { ) } \\
\text { stable (asymptotically for } n=0 \text { ) }\end{array}$ \\
\hline$G$ & $-\frac{\sqrt{6}}{2 \lambda_{V}}$ & $\frac{\sqrt{6}}{2 \lambda_{V}}$ & 0 & 0 & $\lambda_{V}^{2}>3$ & $\begin{array}{c}\text { stable node for } 3<\lambda_{V}^{2}<\frac{24}{7} \\
\text { stable spiral for } \frac{24}{7}<\lambda_{V}^{2}\end{array}$ \\
\hline$O_{2}$ & 0 & 0 & 0 & 0 & always & saddle \\
\hline
\end{tabular}

Table 4. Scenario 2: Exponential potential and power-law coupling function. The real and physically meaningful critical points of the autonomous system (3.24)-(3.27), and their existence and stability conditions.

that the stability of the above points does not depend on the exponent $n$, since these points have $z_{c}=0$ in which case $n$ disappears from the equations and $v$ decouples. Similarly, since they correspond to $v=0$, that is to $\phi \rightarrow \infty$, their coordinates themselves do not depend on $n$ (in other words for $\phi \rightarrow \infty$ all exponents $n \neq 0$, of the same sign, are equivalent). 


\begin{tabular}{|c|c|c|c|c|c|c|}
\hline Cr. P. & $\Omega_{D E}$ & $w_{D E}$ & $w_{t o t}$ & $\mathrm{q}$ & $c_{S}^{2}$ & $Q_{S}$ \\
\hline \hline$E^{ \pm}$ & 1 & 1 & 1 & 2 & 1 & 3 \\
\hline$F$ & 1 & $-1+\frac{\lambda_{V}^{2}}{3}$ & $-1+\frac{\lambda_{V}^{2}}{3}$ & $-1+\frac{\lambda_{V}^{2}}{2}$ & 1 & $\frac{\lambda_{V}^{2}}{2}$ \\
\hline$F_{0}$ & 1 & -1 & -1 & -1 & 1 & 0 \\
\hline$G$ & $\frac{3}{\lambda_{V}^{2}}$ & 0 & 0 & $\frac{1}{2}$ & 1 & $\frac{9}{2 \lambda_{V}^{2}}$ \\
\hline$O_{2}$ & 0 & arbitrary & 0 & $\frac{1}{2}$ & 1 & 0 \\
\hline
\end{tabular}

Table 5. Scenario 2: Exponential potential and power-law coupling function. The real and physically meaningful critical points of the autonomous system (3.24)-(3.27), and the corresponding values of the dark-energy density parameter $\Omega_{D E}$, of the dark-energy equation-of-state parameter $w_{D E}$, of the total equation-of-state parameter $w_{t o t}$ and of the deceleration parameter $q$. The last two columns contain the instability-related parameters $c_{S}^{2}$ and $Q_{S}$ (from (3.32),(3.33)), which must respectively be non-negative and positive for a scenario free of ghosts and instabilities.

\subsubsection{Phase-space analysis at infinity}

Due to the fact that the dynamical system (3.24)-(3.27) is non-compact, there could be features in the asymptotic regime which are non-trivial for the global dynamics. Thus, in order to complete the analysis of the phase space we must extend our study using the Poincaré central projection method [117].

We consider the Poincaré variables

$$
x_{r}=\rho \cos \theta \sin \Phi \sin \psi, y_{r}=\rho \cos \psi, z_{r}=\rho \sin \theta \sin \Phi \sin \psi, v_{r}=\rho \cos \Phi \sin \psi,
$$

where $\rho=\frac{r}{\sqrt{1+r^{2}}}, r=\sqrt{x^{2}+y^{2}+z^{2}+v^{2}}, \theta, \Phi \in[0,2 \pi]$, and $-\frac{\pi}{2} \leq \psi \leq \frac{\pi}{2}$ (we restrict the angle $\psi$ to this interval since the physical region is given by $y>0$ ) [117-120]. Thus, the points at infinity $(r \rightarrow+\infty)$ are those having $\rho \rightarrow 1$. Furthermore, the physical phase space is given by $\left(x_{r}, y_{r}, z_{r}, v_{r}\right) \in[-1,1] \times[0,1] \times[-1,1] \times[-1,1]$, such that

$$
\frac{x_{r}^{2}+y_{r}^{2}}{1-v_{r}^{2}-x_{r}^{2}-y_{r}^{2}-z_{r}^{2}}-\frac{x_{r}^{2} z_{r}\left(\sqrt{6} n v_{r} x_{r}-6 v_{r}^{2}-6 x_{r}^{2}-6 y_{r}^{2}-6 z_{r}^{2}+6\right)}{\left(1-v_{r}^{2}-x_{r}^{2}-y_{r}^{2}-z_{r}^{2}\right)^{5 / 2}} \leq 1,
$$

and $v_{r}^{2}+x_{r}^{2}+y_{r}^{2}+z_{r}^{2} \leq 1$.

Inverting relations (3.34) and substituting into (3.28),(3.29), we obtain the dark energy density and equation-of-state parameters as a function of the Poincaré variables, namely:

$$
\Omega_{D E}=\frac{x_{r}^{2}+y_{r}^{2}}{1-v_{r}^{2}-x_{r}^{2}-y_{r}^{2}-z_{r}^{2}}-\frac{x_{r}^{2} z_{r}\left(\sqrt{6} n v_{r} x_{r}-6 v_{r}^{2}-6 x_{r}^{2}-6 y_{r}^{2}-6 z_{r}^{2}+6\right)}{\left(1-v_{r}^{2}-x_{r}^{2}-y_{r}^{2}-z_{r}^{2}\right)^{5 / 2}},
$$




$$
\begin{gathered}
w_{D E}=\left\{\left[2 z_{r} \sqrt{\zeta}\left(\sqrt{6} n v_{r} x_{r}-3 \zeta\right)+9 x_{r}^{2} z_{r}^{2}+\zeta^{2}\right]\left[\sqrt{6} n v_{r} x_{r}^{3} z_{r}+\zeta^{3 / 2}\left(x_{r}^{2}+y_{r}^{2}\right)-6 x_{r}^{2} z_{r} \zeta\right]\right\}^{-1} \\
\sqrt{\zeta}\left[6 n(n+1) v_{r}^{2} x_{r}^{4} z_{r}^{2}-6 \sqrt{6} n v_{r} x_{r}^{3} z_{r}^{2} \zeta+\sqrt{6} n v_{r} x_{r} z_{r} \zeta^{3 / 2}\left(3 x_{r}^{2}-2 y_{r}^{2}\right)\right. \\
\left.+6 z_{r} \zeta^{5 / 2}\left(y_{r}^{2}-2 x_{r}^{2}\right)+x_{r} z_{r} \zeta^{2}\left(9 x_{r} z_{r}-\sqrt{6} y_{r}^{2} \lambda\right)+\zeta^{3}\left(x_{r}^{2}-y_{r}^{2}\right)\right],
\end{gathered}
$$

where $\zeta=1-x_{r}^{2}-y_{r}^{2}-z_{r}^{2}-v_{r}^{2}$, and similarly substituting into (3.30), (3.31) we obtain the corresponding expressions for the total equation-of-state and deceleration parameters:

$$
\begin{gathered}
w_{\text {tot }}=\left[\zeta^{2}\left(2 \sqrt{6} \sqrt{\zeta} n v_{r} x_{r} z_{r}+9 x_{r}^{2} z_{r}^{2}-6 z_{r} \zeta^{3 / 2}+\zeta^{2}\right)\right]^{-1}\left[6 n(n+1) v_{r}^{2} x_{r}^{4} z_{r}^{2}\right. \\
-6 \sqrt{6} n v_{r} x_{r}^{3} z_{r}^{2} \zeta+\sqrt{6} n v_{r} x_{r} z_{r} \zeta^{3 / 2}\left(3 x_{r}^{2}-2 y_{r}^{2}\right)+6 z_{r} \zeta^{5 / 2}\left(y_{r}^{2}-2 x_{r}^{2}\right) \\
\left.+x_{r} z_{r} \zeta^{2}\left(9 x_{r} z_{r}-\sqrt{6} y_{r}^{2} \lambda\right)+\zeta^{3}\left(x_{r}^{2}-y_{r}^{2}\right)\right], \\
q=\left\{2 \zeta^{2}\left[2 z_{r} \sqrt{\zeta}\left(\sqrt{6} n v_{r} x_{r}-3 \zeta\right)+9 x_{r}^{2} z_{r}^{2}+\zeta^{2}\right]\right\}^{-1}\left\{18 n(n+1) v_{r}^{2} x_{r}^{4} z_{r}^{2}\right. \\
+9 \sqrt{6} n v_{r} x_{r}^{3} z_{r} \zeta\left(\sqrt{\zeta}-2 z_{r}\right)+\sqrt{6} x_{r} z_{r} \zeta^{3 / 2}\left[2 n v_{r}\left(\zeta-3 y_{r}^{2}\right)-3 y_{r}^{2} \sqrt{\zeta} \lambda\right] \\
\left.+3 x_{r}^{2} \zeta^{2}\left(12 z_{r}^{2}-12 z_{r} \sqrt{\zeta}+\zeta\right)+\zeta^{5 / 2}\left(\zeta-3 y_{r}^{2}\right)\left(\sqrt{\zeta}-6 z_{r}\right)\right\} .
\end{gathered}
$$

Finally, substitution into (3.32),(3.33) provides the corresponding expressions for the instabilityrelated quantities:

$$
\begin{gathered}
c_{S}^{2}=\left\{x_{r}\left[2 z_{r} \sqrt{\zeta}\left(\sqrt{6} n v_{r} x_{r}-3 \zeta\right)+9 x_{r}^{2} z_{r}^{2}+\zeta^{2}\right]^{2}\right\}^{-1}\left\{2 \sqrt{6} n v_{r} x_{r}^{2} z_{r} \zeta^{2}\left(\sqrt{\zeta}-4 z_{r}\right)\right. \\
3 x_{r}^{3} z_{r}^{2} \zeta\left[2(n-1) n v_{r}^{2}-6 z_{r} \sqrt{\zeta}+5 \zeta\right]+3 \sqrt{6} n v_{r} x_{r}^{4} z_{r}^{3} \sqrt{\zeta}-27 x_{r}^{5} z_{r}^{4} \\
\left.+x_{r} \zeta^{2}\left[\zeta\left(15 z_{r}^{2}-4 z_{r} \sqrt{\zeta}+\zeta\right)-9 y_{r}^{2} z_{r}^{2}\right]+\sqrt{6} y_{r}^{2} z_{r} \zeta^{3} \lambda\right\} \\
Q_{S}=\frac{3 x_{r}^{2}\left[2 z_{r} \sqrt{\zeta}\left(\sqrt{6} n v_{r} x_{r}-3 \zeta\right)+9 x_{r}^{2} z_{r}^{2}+\zeta^{2}\right]}{\left(3 x_{r}^{2} z_{r}+\zeta^{3 / 2}\right)^{2}}
\end{gathered}
$$

\begin{tabular}{|c|c|c|c|c|c|c|c|c|c|c|c|}
\hline Cr. P. & $x_{r}$ & $y_{r}$ & $z_{r}$ & $v_{r}$ & Stability & $\Omega_{D E}$ & $w_{D E}$ & $w_{t o t}$ & $\mathrm{q}$ & $c_{S}^{2}$ & $Q_{S}$ \\
\hline \hline$L^{ \pm}$ & 0 & 0 & \pm 1 & 0 & unstable & 0 & arbitrary & 0 & $\frac{1}{2}$ & arbitrary & 0 \\
\hline$M^{ \pm}$ & 0 & 0 & 0 & \pm 1 & saddle point & 0 & 1 & 0 & $\frac{1}{2}$ & 1 & 0 \\
\hline
\end{tabular}

Table 6. Scenario 2: Exponential potential and power-law coupling function. The real and physically meaningful critical points at infinity of the autonomous system (3.24)-(3.27), and the corresponding values of the dark-energy density parameter $\Omega_{D E}$, of the dark-energy equation-of-state parameter $w_{D E}$, of the total equation-of-state parameter $w_{t o t}$ and of the deceleration parameter $q$. The last two columns contain the instability-related parameters $c_{S}^{2}$ and $Q_{S}$ (from $(3.40),(3.41)$ ), which must respectively be non-negative and positive for a scenario free of ghosts and instabilities.

Therefore, performing the analysis described in Appendix B.2 we are led to the critical points at infinity $L^{ \pm}$and $M^{ \pm}$, which are presented in Table 6 along with their stability conditions. 


\section{Cosmological Implications}

Since we have performed the complete phase-space analysis of a quite general subclass of generalized Galileon scenario, we can now discuss the corresponding cosmological behavior. In the following subsections we analyze the two different scenarios, namely that of exponential potential and exponential coupling function, and that of exponential potential and power-law coupling function, separately. In each scenario we analyze the observables at the solutions, and moreover the instability-related quantities $c_{S}$ and $Q_{S}$, examining whether they satisfy $(2.24),(2.25)$ in order for the corresponding solution to be physical. However, we mention that this ghost investigation is applied at the critical points, thus even if they are found to be ghost and instabilities free, it is not a proof that the universe evolution towards them did not pass through a state with ghosts and Laplacian instabilities. Therefore in order to obtain a physical evolution of the universe one has to ensure that conditions $(2.24),(2.25)$ are satisfied everywhere in the examined evolution, and not only at late times.

\subsection{Scenario 1: Exponential potential and exponential coupling function}

The critical points of the scenario at hand are the following. Points $A^{ \pm}$exist always, that is for every values of the scenario parameters $\lambda_{V}, \lambda_{g}, V_{0}, g_{0}$, they are unstable or saddle, and thus they cannot be the late-time state of the universe. They correspond to a nonaccelerating, dark-energy dominated universe, with a stiff dark-energy equation-of-state parameter equal to 1 . Finally, the instability-related quantities $c_{S}$ and $Q_{S}$ do satisfy the corresponding conditions $(2.24),(2.25)$, namely $c_{S} \geq 0$ and $Q_{S}>0$, and thus these solutions are free of instabilities. Both of them exist in standard quintessence [107].

Point $O_{1}$ is a saddle one and thus it cannot attract the universe at late times. It corresponds to a non-accelerating, dark-matter dominated universe, with zero total equationof-state parameter. The instability-related quantities $c_{S}$ and $Q_{S}$ satisfy $(2.24),(2.25)$, and thus this solution is free of instabilities (the fact that $Q_{S}$ is exactly 0 and not positive is not a problem, since this happens only at one point and it is not zero identically, in which case there might be a non-perturbative ghost $\left.[121,122]^{2}\right)$. This point exists in standard quintessence too [107].

Point $C$ exists for $0<\lambda_{V}^{2}<6$ and it is a stable one in the region of the parameter space shown in Table 1, and thus it can attract the universe at late times. It corresponds to a dark-energy dominated universe, with a dark-energy equation-of-state parameter lying in the quintessence regime, which can be accelerating or not according to the $\lambda_{V}$-value. Additionally, this solution is free of instabilities. This point exists in standard quintessence [107]. It is quite important, since it is both stable and possesses $w_{D E}$ and $q$ compatible with observations.

Furthermore, we mention that in the specific case where $\lambda_{V}=0$, that is in the case of constant or zero usual potential, there exist the critical point $C_{0}$, and it is always stable. It corresponds to the de Sitter solution, where the universe is accelerating and dark-energy dominated, with the dark-energy behaving like a cosmological constant $\left(w_{D E}=-1\right)$, and it is free of instabilities. This point exists in standard quintessence [107]. It is quite

\footnotetext{
${ }^{2}$ We thank A. De Felice for this comment.
} 
important, since it is both stable and possesses $w_{D E}$ and $q$ compatible with observations. Although at first sight it seems to be the $\lambda_{V} \rightarrow 0$ limit of point $C$, this is not the case since the eigenvalues of $C_{0}$ do not arise from the $\lambda_{V} \rightarrow 0$ limit of $C$-eigenvalues $\left(\lambda_{V}=0\right.$ is a bifurcation value) and thus it has to be considered separately. Moreover, since in many Galileon works the authors do not consider a potential, point $C_{0}$ just gives the late-time state of the universe in these cases $[18,62]$.

Point $D$ exists for $\lambda_{V}^{2} \geq 3$ and in this case it is always stable, that is it can be the late-time state of the universe, and it is free of instabilities. It has the advantage that the dark-energy density parameter is in the interval $0<\Omega_{D E}<1$, that is it can alleviate the coincidence problem since dark energy and dark matter density parameters can be of the same order. However, it has the disadvantage that it is not accelerating and $w_{D E}=0$, which are not favored by observations. This point exists in standard quintessence [107] too.

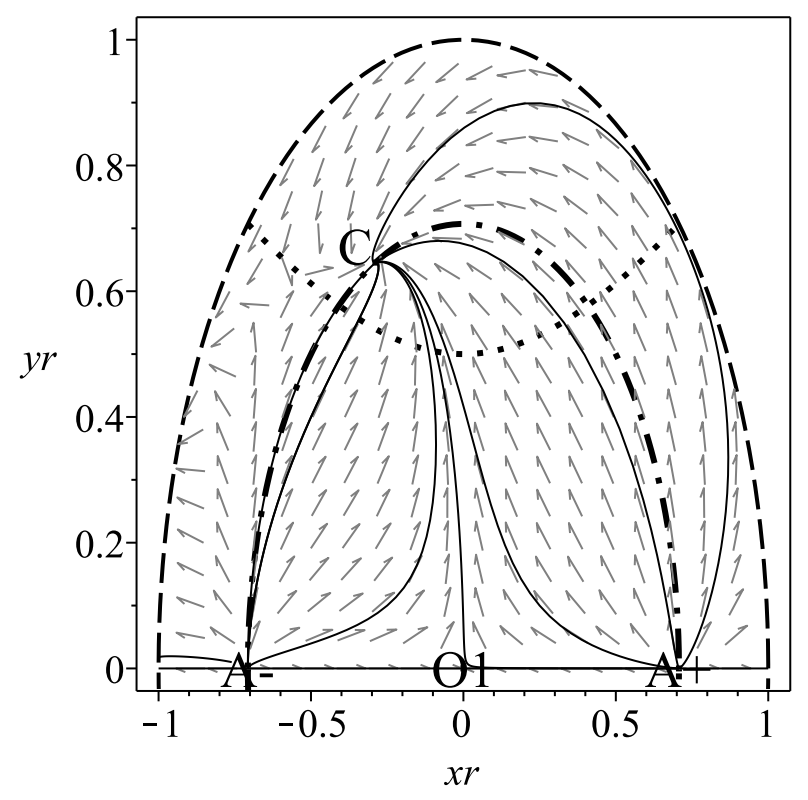

Figure 1. Trajectories in the $y_{r}-x_{r}$ plane of the Poincaré phase space for the Scenario 1, that is for exponential potential and exponential coupling function. We use $\lambda_{V}=1$ and $\lambda_{g}$ arbitrary (for the numerics we choose $\lambda_{g}=1$ but different $\lambda_{g}$ 's correspond to the same projection on $y_{r}-x_{r}$ plane). The region inside the inner semi-circle (seen as semi-ellipse in the figure scale), marked by the thick dashed-dotted line, is the physical part of the phase space. The region above the dotted line marks the region corresponding to accelerating universe $(q<0)$. In this projection the dark-energy dominated, accelerating, quintessence-like solution $C$ is a stable solution, $O_{1}$ is saddle point, and $A^{ \pm}$are unstable.

Apart from the above points that exist also in standard quintessence, the scenario at hand possesses two additional critical points, namely $B^{ \pm}$. They correspond to dark-energy domination, with a dark-energy equation-of-state parameter lying in the quintessence regime, where the universe is non-accelerating $(q>0)$, and they are free of instabili- 
ties. However, these points are not stable and thus they cannot attract the universe at late times.

Finally, the present scenario possesses two critical point at infinity, namely $K^{ \pm}$. They correspond to a dark-matter dominated, non-accelerating universe, with arbitrary $w_{D E}$ but with a zero total equation-of-state parameter $w_{t o t}$, which are also free of instabilities. They are always unstable and therefore they cannot be the late-time state of the universe.

From the above analysis we observe that at the stable critical points, $C$ and $D$, we have $\dot{\phi} \rightarrow 0, \phi \rightarrow-\operatorname{sign}\left(\lambda_{V}\right) \infty$ and thus for $\lambda_{V} \lambda_{g}>0$ we obtain $g(\phi) \rightarrow 0$ while for $\lambda_{V} \lambda_{g}<0$ we obtain $g(\phi) \rightarrow \infty$ (for $\lambda_{V}=0, g(\phi)$ can be zero, finite, or infinity). Similarly, for $C_{0}$ we see that for $\lambda_{g}>0$ we obtain $g(\phi) \rightarrow 0$ while for $\lambda_{g}<0$ we obtain $g(\phi) \rightarrow \infty$. In all cases, if $\lambda_{g}=0$ then obviously $g(\phi)=$ const. From these we deduce that Galileons (simple or generalized) may survive at late-time cosmology or may be completely disappeared by the dynamics, depending on the model parameters. However, firstly we observe that Galileon cosmology possesses the same stable late-time solutions with standard quintessence [107]. Secondly, and more interestingly, as we observe from Table 2, the corresponding observables of these solutions do not depend on the Galileon terms, but only on the usual terms and especially on the standard scalar potential (note that even the instability-related quantities do not depend on the Galileon terms either). This is a main result of the present work.

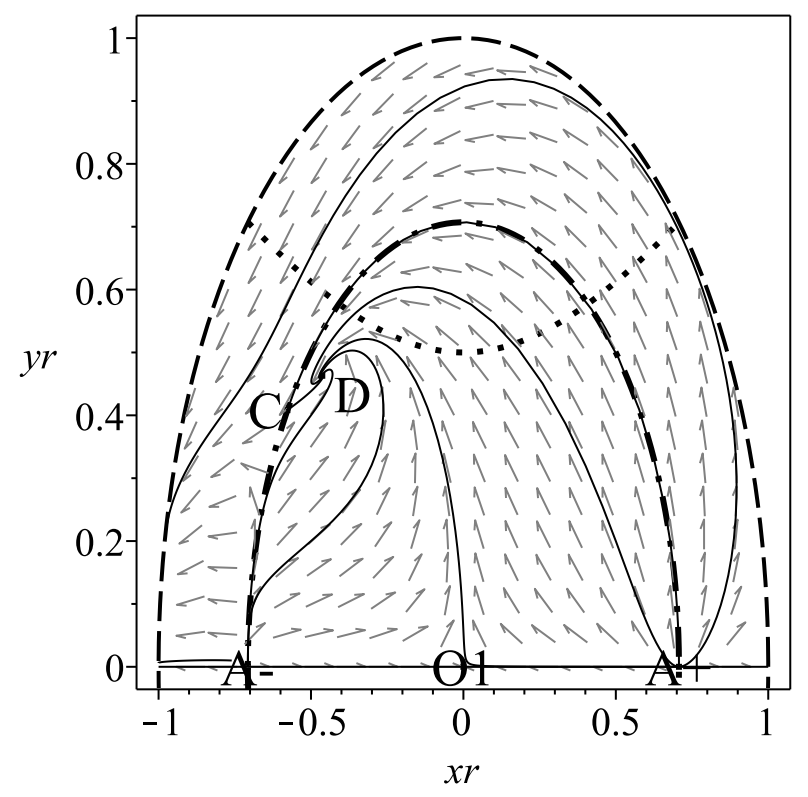

Figure 2. Trajectories in the $y_{r}-x_{r}$ plane of the Poincaré phase space for the Scenario 1, that is for exponential potential and exponential coupling function. We use $\lambda_{V}=2$ and $\lambda_{g}$ arbitrary (for the numerics we choose $\lambda_{g}=1$ but different $\lambda_{g}$ 's correspond to the same projection on $y_{r}-x_{r}$ plane). The region inside the inner semi-circle (seen as semi-ellipse in the figure scale), marked by the thick dashed-dotted line, is the physical part of the phase space. The region above the dotted line marks the region corresponding to accelerating universe $(q<0)$. In this projection the non-accelerating, dust-like $\left(w_{D E}=0\right)$ solution $D$ is a stable spiral, $C$ and $O_{1}$ are saddle points, and $A^{ \pm}$are unstable. 
Therefore, although the Galileon terms of generalized Galileon cosmology can play an important role at early (inflationary) times [28-35] or at recent times [18-27], in the future they will not have any effect on the universe evolution, when it will asymptotically reach its stable state. This is consistent with observational constraints, which disfavor the Galileon presence in the current universe [43, 48, 66-68, 90] (see also [70]). Finally, one could still ask whether going beyond the background analysis, and examining perturbationrelated observables at the late-time stable solutions, would reveal a dependence of these observables on the Galileons. Although this complicated project lies beyond the scope of the present work, the examination of the instability-related quantities $c_{S}$ and $Q_{S}$, which arise from a perturbative analysis, and their independence on the Galileon terms, is an indication that even the perturbation observables will not depend on the Galileon terms.

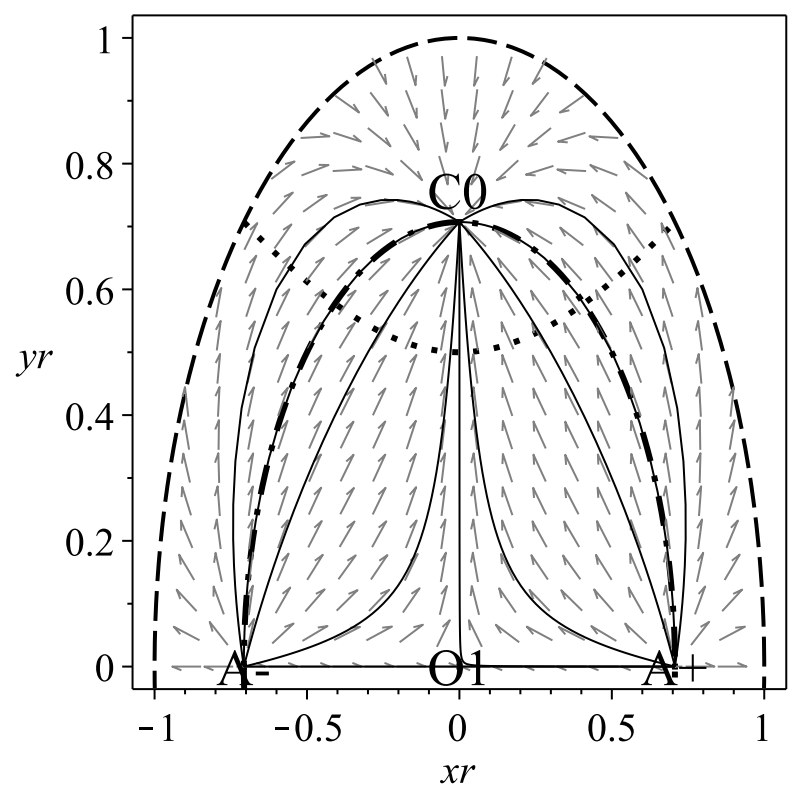

Figure 3. Trajectories in the $y_{r}-x_{r}$ plane of the Poincaré phase space for the Scenario 1, that is for exponential potential and exponential coupling function, for the specific case $\lambda_{V}=0$ and $\lambda_{g}$ arbitrary (for the numerics we choose $\lambda_{g}=1$ but different $\lambda_{g}$ 's correspond to the same projection on $y_{r}-x_{r}$ plane). The region inside the inner semi-circle (seen as semi-ellipse in the figure scale), marked by the thick dashed-dotted line, is the physical part of the phase space. The region above the dotted line marks the region corresponding to accelerating universe $(q<0)$. In this projection the de Sitter solution $C_{0}$ is a stable node, $O_{1}$ is saddle point, and $A^{ \pm}$are unstable.

In order to present the above behavior in a more transparent way, we first evolve the autonomous system (3.7)-(3.9) numerically for the choice $\lambda_{V}=1$ and $\lambda_{g}$ arbitrary (with $\left.\lambda_{g}>-\lambda_{V}\right)$, and in Fig. 1 we depict the corresponding phase-space behavior, projected in the Poincaré $x_{r}-y_{r}$ plane (for the numerics we choose $\lambda_{g}=1$ but different $\lambda_{g}$ 's correspond to the same projection on $y_{r}-x_{r}$ plane). As we can see, in this case the dark-energy dominated, accelerating, quintessence-like critical point $C$ is the late-time solution of the universe.

Similarly, in Fig. 2 we depict the corresponding phase-space behavior, for $\lambda_{V}=2$ and 
$\lambda_{g}$ arbitrary (we use $\lambda_{g}=1$, which satisfies $\lambda_{g}>-\lambda_{V}$ ). In this case the non-accelerating critical point $D$ is the late-time solution of the universe, while $C$ is saddle point. Finally, in Fig. 3 we depict the phase-space behavior for the specific case $\lambda_{V}=0$ and $\lambda_{g}$ arbitrary. In this case the universe is attracted by the de Sitter solution $C_{0}$ as expected.

\subsection{Scenario 2: Exponential potential and power-law coupling function}

This scenario possesses the following critical points. $E^{ \pm}$exist for every value of the parameters $\lambda_{V}, n, V_{0}, g_{0}$, they are saddle points, and thus they cannot be the late-time state of the universe. They correspond to a non-accelerating, dark-energy dominated universe, with a stiff dark-energy equation-of-state parameter equal to 1 , and they are free of instabilities. Both of them exist in standard quintessence [107].

Point $\mathrm{O}_{2}$ is a saddle one and thus it cannot attract the universe at late times. It corresponds to a non-accelerating, dark-matter dominated universe, with zero total equationof-state parameter, and it is free of instabilities. This point exists in standard quintessence too [107].

Point $F$ exists for $0<\lambda_{V}^{2}<6$ and it is a stable one for $\lambda_{V}^{2}<3$ and thus it can be the late-time state of the universe. It corresponds to a dark-energy dominated universe, with a dark-energy equation-of-state parameter in the quintessence regime, which can be accelerating or not according to the $\lambda_{V}$-value. Additionally, this solution is free of instabilities. This point is quite important, since it is both stable and possesses $w_{D E}$ and $q$ compatible with observations. It exists in standard quintessence [107] too.

There is another interesting critical point, namely $F_{0}$, which is obtained only in the case where $\lambda_{V}=0$, that is in the case of constant or zero usual potential, and it is always stable. It corresponds to the de Sitter solution, where the universe is accelerating and dark-energy dominated, with the dark-energy behaving like a cosmological constant $\left(w_{D E}=-1\right)$, and it is free of instabilities. At first sight it seems to be the $\lambda_{V} \rightarrow 0$ limit of point $F$, however this is not the case since the eigenvalues of $F_{0}$ do not arise from the $\lambda_{V} \rightarrow 0$ limit of $F$-eigenvalues ( $\lambda_{V}=0$ is a bifurcation value) and thus it has to be considered separately. Furthermore, since in many Galileon works the standard potential is not considered, point $F_{0}$ is straightforwardly the corresponding late-time state of the universe in these cases.

Point $G$ exists for $\lambda_{V}^{2} \geq 3$ and in this case it is always stable, that is it can attract the universe at late times, and it is free of instabilities. It has the advantage that the dark-energy density parameter lies in the interval $0<\Omega_{D E}<1$, that is it can alleviate the coincidence problem, but it has the disadvantage that it is not accelerating and possesses $w_{D E}=0$, which are not favored by observations. This point exists in standard quintessence [107] too.

Finally, the scenario at hand possesses the critical point at infinity $L^{ \pm}$and $M^{ \pm}$. They correspond to a dark-matter dominated, non-accelerating universe, with a zero total equation-of-state parameter $w_{t o t}$, which are also free of instabilities. $M^{ \pm}$are saddle points whereas $L^{ \pm}$are unstable, and thus they cannot be the late-time state of the universe.

¿From the above analysis we can see that at the stable critical points, $F$ and $G$, we have $\dot{\phi} \rightarrow 0, \phi \rightarrow-\operatorname{sign}\left(\lambda_{V}\right) \infty$ and thus for $n<0$ we obtain $g(\phi) \rightarrow 0$ while for $n>0$ we obtain $g(\phi) \rightarrow \infty$. Similarly, for $F_{0}$ we see that for $n<0$ we obtain $g(\phi) \rightarrow 0$ while for $n>0$ 
we obtain $g(\phi) \rightarrow \infty$. In all cases, if $n=0$ then obviously $g(\phi)=$ const. From these it is implied that Galileons (simple or generalized) may survive at late-time cosmology or may be completely disappeared by the dynamics, depending on the model parameters. Similarly to the previous subsection, firstly we observe that this scenario possesses the same stable late-time solutions with standard quintessence [107]. Furthermore, as we observe from Table 5, the corresponding observables of these solutions do not depend on the Galileon terms, but only on the usual terms and especially on the standard scalar potential. Even the instability-related quantities do not depend on the Galileon terms either. This is a main result of the present work.

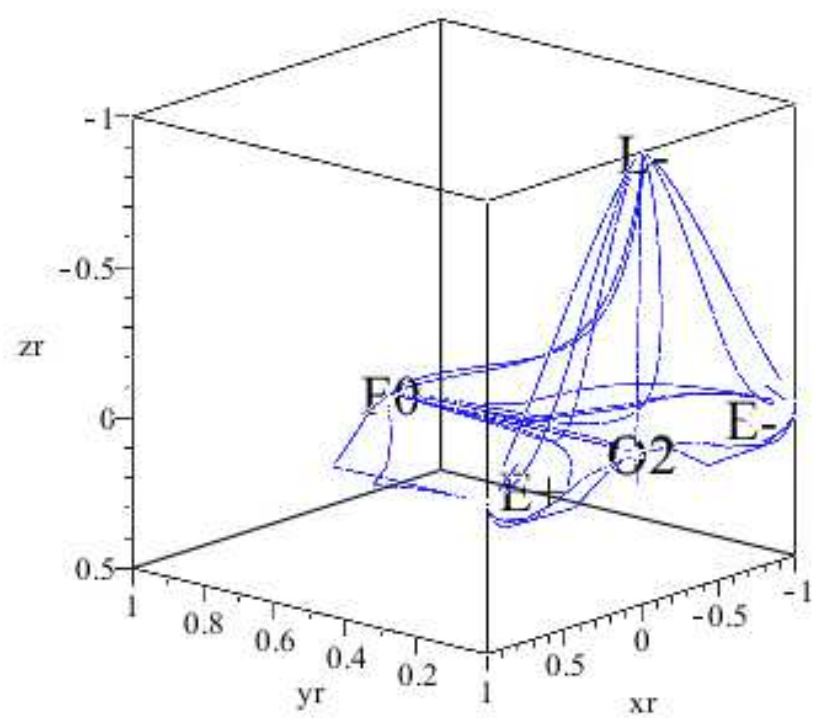

Figure 4. Projection of orbits on the $x_{r}-y_{r}-z_{r}$ space of the Poincaré phase space for the Scenario 2, that is for exponential potential and power-law coupling function, for the specific case $\lambda_{V}=0$ and $n$ arbitrary (for the numerics we choose $n=1$ but different $n$ 's correspond to the same projection). In this projection the de Sitter solution $F_{0}$ is the attractor, whereas $L^{-}$and $E^{ \pm}$are unstable and $\mathrm{O}_{2}$ is a saddle point.

Thus, and similarly to the scenario of the previous subsection, although the Galileons can play an important role at inflationary [28-35] or at recent times [18-27], in the future, when the universe will asymptotically reach its stable state, they will not have any effect on its evolution (this is consistent with observational constraints which disfavor the Galileon presence in the current universe [43, 48, 66-68, 90]. One could still ask whether the observables related to perturbations would depend on the Galileon terms, however the investigation of the instability-related quantities $c_{S}$ and $Q_{S}$, which come from a perturba- 
tive analysis, and their independence on the Galileon terms, is an indication that even the perturbation observables will not depend on the Galileon terms.

In order to present the above behavior in a more transparent way, we first evolve the autonomous system (3.24)-(3.27) numerically for the choice $\lambda_{V}=0$ and $n$ arbitrary, and in Fig. 4 we depict the corresponding phase-space behavior, projected on the Poincaré $x_{r}-y_{r}-z_{r}$ space (for the numerics we choose $n=1$ but different $n$ 's correspond to the same projection). As we can wee, in this case the de Sitter critical point $F_{0}$ is the late-time solution of the universe while $L^{-}$and $E^{ \pm}$are unstable. Finally, note that the sign of $z_{r}$ is invariant, thus the plane $z_{r}=0$ cannot be crossed by the orbits.

We close this section by comparing the present scenario of exponential potential and power-law coupling function, with the one of the previous subsection, that is with exponential potential and exponential coupling function. A first observation is that for $\lambda_{g}=0$ and $n=0$ the critical points of both scenarios coincide, which was expected since in this case the two models coincide and they both transit to the simple Galileon scenario, where the couplings are constants and not functions of the scalar field. Furthermore, in both scenarios we found that there are not additional stable late-time solutions apart from those of standard quintessence. What is more interesting is that all the observables do not depend on the Galileon terms, that is the Galileons do not play any role at late times. However, in general the two scenarios are different, with the main difference being that they have different non-stable critical points and thus phase-space dynamics.

\section{Conclusions}

In the present work we have investigated the dynamical behavior of generalized Galileon cosmology, which is a recent construction based on higher derivatives in the action, along with the imposition of the Galilean symmetry, with the requirement of maintaining the equations of motion second ordered. However, one additionally extends the constant coefficients of the various action-terms of the simple Galileon formulation, into arbitrary functions of the scalar field.

Performing a detailed phase-space analysis of two distinct and quite general scenarios, namely of exponential usual potential and exponential Galileon coupling-function, and exponential usual potential and power-law Galileon coupling-function, we extracted the stable solutions, that is the solutions that will be the late-time states of the universe. In each of these stable late-time solutions we have calculated various observables, such as the dark-energy equation-of-state parameter, the deceleration parameter, and the darkenergy and matter density parameters. Furthermore, in order to examine whether these solutions are free of ghosts and instabilities, we calculate the corresponding instabilityrelated quantities of the literature.

In the case where the Galileon terms are absent we recovered the results of standard quintessence. In the case where the Galileons are present we found that at late times they may survive at or they may completely disappear by the dynamics, depending on the model parameters. However, independently of their disappearance or survival, we found that the scenario at hand possesses exactly the same stable late-time solutions with standard 
quintessence, which are moreover free of ghosts and instabilities. More interestingly, the corresponding observables at these stable late-time solutions do not depend on the Galileon terms, but only on the usual action-terms and especially on the standard scalar potential. This is a main result of the present work, and it shows that although the Galileons can play an important role at inflationary [28-35] or at recent times [18-27], in the future, when the universe will asymptotically reach its stable state, they will not have any effect on its evolution. This is in agreement with observational constraints which disfavor the Galileon presence in the current universe [43, 48, 66-68, 90].

One could ask whether the above behavior is a result of the specific ansantzes, or it has a general character. Although one cannot exclude the case where suitably chosen or tuned ansatzes can lead to significant Galileon effects on the observables at late times, in general one expects the above behavior to be valid at late times in the large majority of cases, due to the downgrading of the role of the derivative (Galileon) terms in an eternally expanding universe. However, we mention that in the present work we followed the majority of the Galileon cosmological works and we viewed the Galileon theory as a scalar-field, dark-energy, construction, and not as a modified gravity, and thus we did not include a coupling between the scalar field and the matter sector which existed in the initial Galileon formulation. Such a coupling could indeed lead to significantly different cosmological behavior, and its investigation is left for a future project.

We close this work by mentioning that the obtained result that the Galileons will not play any role and that they will not have any observable effect at late times, was extracted for the background evolution and the corresponding observables. There could still be the case that Galileons could leave their signatures in observables related to perturbations. Although such an investigation is quite complicated and lies beyond the scope of the present work, the examination of instability-related quantities arising from perturbation analysis that we did perform in this work showed that they do not depend on the Galileon terms either, which is a quite strong indication that even the perturbation observables will not depend on the Galileon terms.

\section{Acknowledgments}

The authors wish to thank S. Lepe for fruitful discussions and S. A. Appleby, P. Creminelli, A. De Felice, J. Khoury, E. V. Linder and A. Nicolis, for useful comments. The research project is implemented within the framework of the Action "Supporting Postdoctoral Researchers" of the Operational Program "Education and Lifelong Learning" (Actions Beneficiary: General Secretariat for Research and Technology), and is co-financed by the European Social Fund (ESF) and the Greek State. Additionally, it is co-financed by MECESUP FSM0806 from Ministerio de Educación, Chile and by PUCV through Proyecto DI Postdoctorado 2013. GL wishes to thank to his colleagues at Instituto de Física, Pontificia Universidad de Católica de Valparaíso for their warm hospitality during the completion of this work. 


\begin{tabular}{|c|c|c|c|}
\hline Cr. P. & $\nu_{1}$ & $\nu_{2}$ & $\nu_{3}$ \\
\hline \hline$A^{+}$ & 3 & $\sqrt{6} \lambda_{g}-6$ & $3+\sqrt{\frac{3}{2}} \lambda_{V}$ \\
\hline$A^{-}$ & 3 & $-\sqrt{6} \lambda_{g}-6$ & $3-\sqrt{\frac{3}{2}} \lambda_{V}$ \\
\hline$B^{+}$ & $\frac{3}{4}\left[-\frac{\sqrt{2} \lambda_{g}}{\left.\sqrt{\alpha^{-}\left(\lambda_{g}\right)-3}+\alpha^{+}\left(\lambda_{g}\right)-4\right]}\right.$ & $\frac{3}{4}\left[\frac{\sqrt{2} \lambda_{g}}{\sqrt{\alpha^{-}\left(\lambda_{g}\right)-3}}+\alpha^{+}\left(\lambda_{g}\right)-4\right]$ & $\frac{\left(\lambda_{g}+\lambda_{V}\right) \alpha^{+}\left(\lambda_{g}\right)}{2 \lambda_{g}}$ \\
\hline$B^{-}$ & $\frac{3}{4}\left[-\frac{\sqrt{2} \lambda_{g}}{\left.\sqrt{\alpha^{+}\left(\lambda_{g}\right)-3}+\alpha^{-}\left(\lambda_{g}\right)-4\right]}\right.$ & $\frac{3}{4}\left[\frac{\sqrt{2} \lambda_{g}}{\left.\sqrt{\alpha^{+}\left(\lambda_{g}\right)-3}+\alpha^{-}\left(\lambda_{g}\right)-4\right]}\right.$ & $\frac{\left(\lambda_{g}+\lambda_{V}\right) \alpha^{-}\left(\lambda_{g}\right)}{2 \lambda_{g}}$ \\
\hline$C^{2}$ & $-\lambda_{V}^{2}-3$ & $-\lambda_{V}\left(\lambda_{g}+\lambda_{V}\right)$ & $\frac{1}{2}\left(\lambda_{V}^{2}-6\right)$ \\
\hline$C_{0}$ & $-\frac{3\left(\lambda_{g}+\lambda_{V}\right)}{\lambda_{V}}$ & -3 & -3 \\
\hline$D$ & $-\frac{9}{2}$ & $\left.-\frac{\sqrt{24 \lambda_{V}^{2}-7 \lambda_{V}^{4}}}{2}-1\right)$ & $\frac{3}{4}\left(\frac{\sqrt{24 \lambda_{V}^{2}-7 \lambda_{V}^{4}}}{\lambda_{V}^{2}}-1\right)$ \\
\hline$O_{1}$ & -3 & $\frac{3}{2}$ \\
\hline
\end{tabular}

Table 7. The eigenvalues of the matrix $\mathbf{Q}$ of the perturbation equations of the autonomous system (3.7)-(3.9). We have defined the functions $\alpha^{ \pm}\left(\lambda_{g}\right)=\lambda_{g}^{2} \pm \lambda_{g} \sqrt{\lambda_{g}^{2}-6}$.

\section{A Stability of Scenario 1}

\section{A.1 Stability of the finite critical points}

For the critical points $\left(x_{c}, y_{c}, z_{c}\right)$ of the autonomous system system (3.7)-(3.9), the coefficients of the perturbation equations form a $3 \times 3$ matrix $\mathbf{Q}$, which can be easily obtained, however, since they are rather lengthy expressions we do not present them explicitly. Despite this complicated form, using the specific critical points presented in Table 1 the matrix $\mathbf{Q}$ acquires a simple form that allows for an easy calculation of its eigenvalues. The corresponding eigenvalues $\nu_{i}(i=1,2,3)$ for each critical point are presented in Table 7 .

Thus, by determining the sign of the real parts of these eigenvalues, we can classify the corresponding critical point. In particular, if all the eigenvalues of a critical point have negative real parts then the corresponding point is stable, if they all have positive real parts then it is unstable, and if they change sign then it is a saddle point.

We mention here that in order to examine correctly the stability of point $C_{0}$ (since the second eigenvalue vanishes at first place) we introduce the local coordinates

$$
\{x, y-1, z\}=\epsilon\{\tilde{x}, \tilde{y}, \tilde{z}\}+\mathcal{O}\left(\epsilon^{2}\right)
$$


where $\epsilon$ is a constant satisfying $\epsilon \ll 1$. Then we obtain the local evolution equations

$$
\tilde{x}^{\prime}=-3 \tilde{x}+\text { h.o.t }, \quad \tilde{y}^{\prime}=-3 \tilde{y}+\text { h.o.t }, \quad \tilde{z}^{\prime}=-3 \tilde{z}+\text { h.o.t },
$$

with h.o.t denoting "higher order terms". From the above linear approximation we deduce that the eigenvalues of the linearization for point $C_{0}$ are all negative and are displayed in Table 7 , thus $C_{0}$ is a stable node.

Similarly, in order to examine correctly the stability of point $O_{1}$ we introduce the local coordinates

$$
\{x, y, z\}=\epsilon\{\tilde{x}, \tilde{y}, \tilde{z}\}+\mathcal{O}\left(\epsilon^{2}\right)
$$

with $\epsilon \ll 1$. The local evolution equations valid around the origin then write as

$$
\tilde{x}^{\prime}=-\frac{3}{2} \tilde{x}+\text { h.o.t }, \quad \tilde{y}^{\prime}=\frac{3}{2} \tilde{y}+\text { h.o.t }, \quad \tilde{z}^{\prime}=-\frac{9}{2} \tilde{z}+\text { h.o.t. }
$$

The eigenvalues of the linearization for point $O_{1}$ are displayed in Table 7, and thus $O_{1}$ is a saddle point.

\section{A.2 Stability of the critical points at infinity}

We consider the Poincaré variables

$$
x_{r}=\rho \cos \theta \sin \psi, z_{r}=\rho \sin \theta \sin \psi, y_{r}=\rho \cos \psi,
$$

with $\rho=\frac{r}{\sqrt{1+r^{2}}}, r=\sqrt{x^{2}+y^{2}+z^{2}}, \theta \in[0,2 \pi]$, and $-\frac{\pi}{2} \leq \psi \leq \frac{\pi}{2}$ (we restrict the $\psi$ angle to this range since the physical region is given by $y>0$ ) [117-120]. Therefore, the points at infinity $(r \rightarrow+\infty)$ are those with $\rho \rightarrow 1$. Moreover, the physical phase space is now given by $\left(x_{r}, y_{r}, z_{r}\right) \in[-1,1] \times[0,1] \times[-1,1]$ such that

$$
\frac{x_{r}^{2}+y_{r}^{2}}{1-x_{r}^{2}-y_{r}^{2}-z_{r}^{2}}-\frac{x_{r}^{2} z_{r}\left(\sqrt{6} \lambda_{g} x_{r}-6 x_{r}^{2}-6 y_{r}^{2}-6 z_{r}^{2}+6\right)}{\left(1-x_{r}^{2}-y_{r}^{2}-z_{r}^{2}\right)^{5 / 2}} \leq 1,
$$

and $x_{r}^{2}+y_{r}^{2}+z_{r}^{2} \leq 1$.

Performing the transformation (A.3) on the system (3.7)-(3.9), and taking the limit $\rho \rightarrow 1$, the leading terms in the resulting system are

$$
\begin{aligned}
& \rho^{\prime} \rightarrow-\lambda_{g}^{2} \cos ^{2} \theta \sin ^{2} \psi\left[\cos (2 \theta) \sin ^{2} \psi+\cos ^{2} \psi\right], \\
& \left(1-\rho^{2}\right) \theta^{\prime} \rightarrow-2 \lambda_{g}^{2} \sin \theta \cos ^{3} \theta \sin ^{2} \psi, \\
& \left(1-\rho^{2}\right) \psi^{\prime} \rightarrow-2 \lambda_{g}^{2} \sin ^{2} \theta \cos ^{2} \theta \sin ^{3} \psi \cos \psi .
\end{aligned}
$$

Since the equation for $\rho$ decouples, we only need to investigate the subsystem of the angular variables. Thus, equating (A.6) and (A.7) to zero, we obtain three classes of solutions:

Class A:

$$
x_{r}=\sin \psi, y_{r}=\cos \psi, z_{r}=0,
$$


for some $\psi$. These points lead to a divergence in $\Omega_{D E}$ in (3.18) and thus we deduce that they are unphysical.

\section{Class B:}

$$
x_{r}=0, y_{r}=\cos \psi, z_{r}= \pm \sin \psi,
$$

for some $\psi$. These points correspond to $\theta= \pm \frac{\pi}{2}$. By substituting these values for $x_{r}, y_{r}, z_{r}$ in the evolution equations for $x_{r}^{\prime}, y_{r}^{\prime}, z_{r}^{\prime}$ we obtain that $y_{r}$ must be zero at a fixed point at infinity. Thus, we have the points at infinity given by $K^{ \pm}:\left[x_{r}=0, y_{r}=0, z_{r}= \pm 1\right]$.

\section{Class C:}

$$
x_{r}=0, y_{r}=1, z_{r}=0 .
$$

This point leads to a divergence in $\Omega_{D E}$ in (3.18) and thus it is unphysical.

In summary, the scenario of exponential potential and exponential coupling function has only two critical points at infinity, namely $K^{ \pm}:\left[x_{r}=0, y_{r}=0, z_{r}= \pm 1\right]$. In order to calculate the eigenvalues of the linearization of the dynamical system for $x_{r}, y_{r}, z_{r}$ around $K^{ \pm}$, we proceed as follows: having calculated the linear perturbation matrix $\mathbf{Q}$ we evaluate it at $y_{r}=0$, then we take the limit $x_{r} \rightarrow 0$ and finally we incorporate the lateral limit $z_{r} \rightarrow 1^{-}$(that is from below) for $K^{+}$or $z_{r} \rightarrow-1^{+}$(that is from above) for $K^{-}$. Calculating the eigenvalues of the resulting matrix we obtain that the eigenvalues of the linearization for both $K^{ \pm}$are given by $\left(3, \frac{15}{4}, \frac{9}{2}\right)$. Therefore, we conclude that $K^{ \pm}$are unstable.

\section{B Stability of Scenario 2}

\section{B.1 Stability of the finite critical points}

For the critical points $\left(x_{c}, y_{c}, z_{c}, v_{c}\right)$ of the autonomous system (3.24)-(3.27), the coefficients of the perturbation equations form a $4 \times 4$ matrix $\mathbf{Q}$. The explicit expressions for these coefficients are quite lengthy and for simplicity we do not present them explicitly. However, using the explicit critical points presented in Table 4 we can straightforwardly see that the matrix $\mathbf{Q}$ acquires a simple form that allows for an easy calculation of its eigenvalues. The corresponding eigenvalues $\nu_{i}(i=1,2,3,4)$ for each critical point are presented in Table 8 .

Note that the stability of the above points does not depend on the exponent $n$, since these points have $z_{c}=0$ in which case $n$ disappears from the equations and $v$ decouples. Similarly, since they correspond to $v=0$, that is to $\phi \rightarrow \infty$, their coordinates themselves do not depend on $n$ (in other words for $\phi \rightarrow \infty$ all exponents $n \neq 0$ of the same sign are equivalent).

Strictly speaking, since the eigenvalue of the fourth auxiliary variable $v$ is always zero, the corresponding points are non-hyperbolic, and thus one should apply the center manifold analysis [123] in order to deduce whether it is unstable, saddle or stable. However, since all the above points have $z_{c}=0, v$ is completely decoupled from the autonomous system (3.24)-(3.27). Therefore, one can examine only the three eigenvalues $\nu_{1}, \nu_{2}, \nu_{3}$ displayed in Table 8 , and the results of the stability analysis are presented in Table 4. Finally, in order 


\begin{tabular}{|c|c|c|c|c|}
\hline Cr. P. & $\nu_{1}$ & $\nu_{2}$ & $\nu_{3}$ & $\nu_{4}$ \\
\hline \hline$E^{ \pm}$ & -6 & $3 \pm \frac{\lambda_{V} \sqrt{6}}{2}$ & 3 & 0 \\
\hline & $\lambda_{V}^{2}-3$ & $\frac{\lambda_{V}^{2}}{2}-3$ & $-\lambda_{V}^{2}$ & 0 \\
\hline$F$ & -3 & -3 & -3 & 0 \\
$F_{0}$ & -3 & $\frac{3}{4}\left(-\frac{\sqrt{24 \lambda_{V}^{2}-7 \lambda_{V}^{4}}}{\lambda_{V}^{2}}-1\right)$ & $\frac{3}{4}\left(\frac{\sqrt{24 \lambda_{V}^{2}-7 \lambda_{V}^{4}}}{\lambda_{V}^{2}}-1\right)$ & 0 \\
\hline$G$ & $-\frac{3}{2}$ & $\frac{3}{2}$ & 0 \\
\hline$O_{2}$ & $-\frac{9}{2}$ & & & \\
\hline
\end{tabular}

Table 8. The eigenvalues of the matrix $\mathbf{Q}$ of the perturbation equations of the autonomous system (3.24)-(3.27).

to examine correctly the stability of points $F_{0}$ and $O_{2}$ we use the same linear approximation with that in the end of appendix A.1 (for points $C_{0}$ and $O_{1}$ ), and we deduce that $F_{0}$ is a stable node while $O_{1}$ is saddle, with eigenvalues depicted in Table 8.

We end this appendix with the following comment. The above stability conditions for $F, F_{0}$ and $G$ we extracted using the fact that the $v$-direction is decoupled. However, as we mentioned the complete dynamical picture should be obtained after a full center manifold analysis [123], which is the correct way to handle zero eigenvalues. Therefore, in the following we present this analysis for completeness. Since compact variables are preferable to see the subtleties on the dynamics, we perform the calculations using a Poincaré projection method introducing the variables

$$
x_{r}=\rho \cos \theta \sin \Phi \sin \psi, y_{r}=\rho \cos \psi, z_{r}=\rho \sin \theta \sin \Phi \sin \psi, v_{r}=\rho \cos \Phi \sin \psi,
$$

where $\rho=\frac{r}{\sqrt{1+r^{2}}}, r=\sqrt{x^{2}+y^{2}+z^{2}+v^{2}}, \theta, \Phi \in[0,2 \pi]$, and $-\frac{\pi}{2} \leq \psi \leq \frac{\pi}{2}[117-120]$.

\section{- Center manifold analysis for $F$}

Using the Poincaré coordinates (B.1), the point $F$ transforms to the point with coordinates $x_{r}=-\frac{\lambda_{V}}{2 \sqrt{3}}, y_{r}=\frac{1}{2} \sqrt{2-\frac{\lambda_{V}^{2}}{3}}, z_{r}=0, v_{r}=0$. Introducing the new variables

$$
\begin{aligned}
& u=v_{r}, \\
& v_{1}=z_{r}, \\
& v_{2}=-\frac{1}{6} x_{r} \sqrt{6-\lambda_{V}^{2}} \lambda_{V}+y_{r}\left(2-\frac{\lambda_{V}^{2}}{6}\right)-\frac{z_{r} \sqrt{1-\frac{\lambda_{V}^{2}}{6}} \lambda_{V}^{4}}{6\left(\lambda_{V}^{2}-2\right)}-\sqrt{2-\frac{\lambda_{V}^{2}}{3}}, \\
& v_{3}=\frac{1}{6} x_{r} \sqrt{6-\lambda_{V}^{2}} \lambda_{V}+\frac{1}{6} y_{r}\left(\lambda_{V}^{2}-6\right)+\frac{1}{4} z_{r} \sqrt{1-\frac{\lambda_{V}^{2}}{6}} \lambda_{V}^{2}+\frac{1}{2} \sqrt{2-\frac{\lambda_{V}^{2}}{3}},
\end{aligned}
$$


we deduce that the local invariant center manifold of $F$ is given by the approximated graph

$$
\begin{aligned}
\left\{\left(u, v_{1}, v_{2}, v_{3}\right) \mid v_{1}=\mathcal{O}\left(u^{4}\right), v_{2}\right. & =-\frac{1}{2} \sqrt{2-\frac{\lambda_{V}^{2}}{3}} u^{2}+\mathcal{O}\left(u^{4}\right), \\
v_{3} & \left.=\frac{1}{4} \sqrt{2-\frac{\lambda_{V}^{2}}{3}} u^{2}+\mathcal{O}\left(u^{4}\right),|u|<\delta\right\},
\end{aligned}
$$

where $\delta>0$ is a suitably small positive real number and $\mathcal{O}(4)$ denotes terms of fourth order in the vector norm. Therefore, the dynamics at the center manifold is given by

$$
u^{\prime}=\sqrt{2} u^{2} \lambda_{V},|u|<\delta,
$$

with solution for $u(0)=u_{0}$ given by

$$
u_{1}(\tau)=\frac{u_{0}}{1-\sqrt{2} u_{0} \lambda_{V} \tau} .
$$

Since the origin is an inflection point of the potential $U(u)=-\frac{\sqrt{2}}{3} u^{3} \lambda_{V}$ of the gradient-like equation (B.4), it is implied that $F$ is a saddle point for $\lambda_{V}^{2}<3$, only for perturbations in the direction of $v_{r}$-axis and thus for perturbations in the $v$-axis.

\section{- Center manifold analysis for $F_{0}$}

Using the Poincaré coordinates (B.1), the point $F_{0}$ transforms to the point with coordinates $x_{r}=0, y_{r}=\frac{\sqrt{2}}{2}, z_{r}=0, v_{r}=0$. Proceeding similarly to point $F$ above and introducing the new variables

$$
u_{1}=v_{r}, v_{1}=x_{r}, v_{2}=y_{r}-\frac{\sqrt{2}}{2}, v_{3}=z,
$$

we find that the center manifold is given by the graph

$$
\left\{\left(u_{1}, v_{1}, v_{2}, v_{3}\right): v_{1}=-\frac{u_{1}^{2}}{2 \sqrt{2}}+\mathcal{O}(4), v_{2}=\mathcal{O}(4), v_{3}=\mathcal{O}(4),\left|u_{1}\right|<\delta\right\}
$$

where $\delta$ is a suitably small constant and $\mathcal{O}(4)$ denotes terms of fourth order in the vector norm. The dynamics on the center manifold is governed by the equation $u_{1}^{\prime}=$ $\mathcal{O}(4)$, from which it follows that $F_{0}$ is indeed stable, but for $n \neq 0$ not asymptotically. This behavior is depicted in Fig. 5 .

\section{- Center manifold analysis for $G$}

Using the Poincaré coordinates (B.1), the point $G$ transforms to the point with coordinates $x_{r}=-\frac{\sqrt{\frac{3}{2}}}{\sqrt{\lambda_{V}^{2}+3}}, y_{r}=\frac{\sqrt{\frac{3}{2}}}{\sqrt{\lambda_{V}^{2}+3}}, z_{r}=0, v_{r}=0$. Introducing the new variables

$$
\begin{aligned}
& u=v_{r}, \\
& v_{1}=z_{r},
\end{aligned}
$$




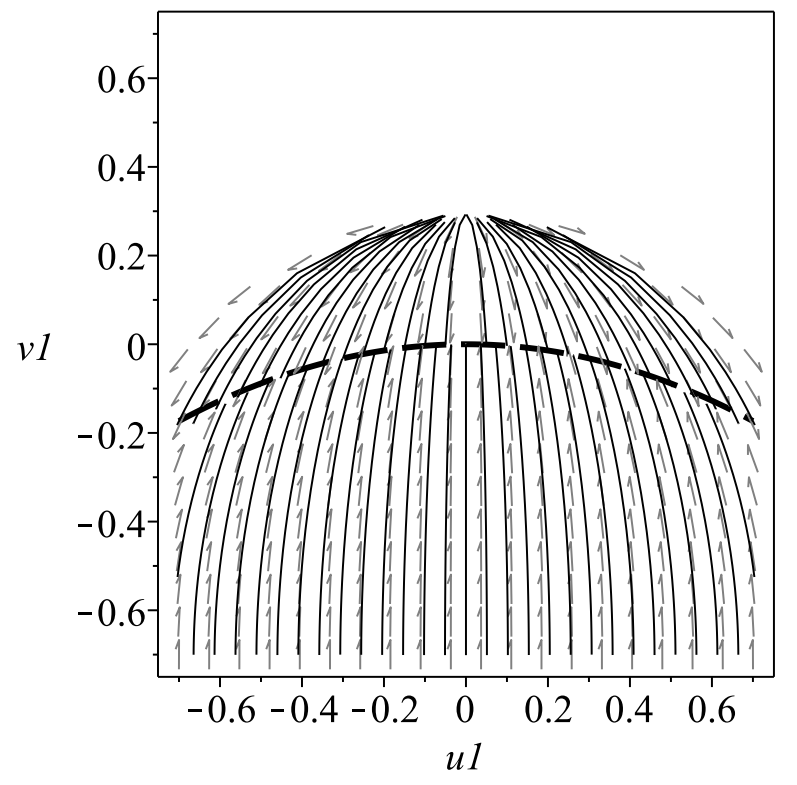

Figure 5. We consider the coordinate system (B.6), using $\lambda_{V}=0$ and $n$ arbitrary (for the numerics we choose $n=1$ but different $n$ 's correspond to the same projection). The dashed thick line represents the center manifold of $F_{0}$, which is stable. The orbits above and below that line tend to it as time goes forward.

$$
\begin{aligned}
v_{2}= & -\frac{x_{r}\left(2 \lambda_{V}^{4}-3 \lambda_{V}^{2}+9\right)}{2 \lambda_{V} \sqrt{24-7 \lambda_{V}^{2}}\left(\lambda_{V}^{2}+3\right)}-\frac{\lambda_{V}\left(\sqrt{24-7 \lambda_{V}^{2}}+\lambda_{V}\right)+6}{2 \lambda_{V} \sqrt{16-\frac{14 \lambda_{V}^{2}}{3}} \sqrt{\lambda_{V}^{2}+3}} \\
+ & \frac{y_{r}\left(-\lambda_{V}^{4}+12 \lambda_{V}^{2}+3 \sqrt{24-7 \lambda_{V}^{2}} \lambda_{V}+\sqrt{24-7 \lambda_{V}^{2}} \lambda_{V}^{3}+9\right)}{2 \lambda_{V} \sqrt{24-7 \lambda_{V}^{2}}\left(\lambda_{V}^{2}+3\right)} \\
- & \frac{3 \sqrt{\frac{3}{2}} z_{r}\left(\sqrt{24-7 \lambda_{V}^{2}}+3 \lambda_{V}\right)\left(2 \lambda_{V}^{4}-3 \lambda_{V}^{2}+9\right)}{8 \lambda_{V} \sqrt{24-7 \lambda_{V}^{2}}\left(\lambda_{V}^{2}+3\right)\left(2 \lambda_{V}^{2}-3\right)}, \\
v_{3}= & \frac{x_{r}\left(2 \lambda_{V}^{4}-3 \lambda_{V}^{2}+9\right)}{2 \lambda_{V} \sqrt{24-7 \lambda_{V}^{2}}\left(\lambda_{V}^{2}+3\right)}+\frac{\lambda_{V}^{2}-\sqrt{24-7 \lambda_{V}^{2}} \lambda_{V}+6}{2 \lambda_{V} \sqrt{16-\frac{14 \lambda_{V}^{2}}{3}} \sqrt{\lambda_{V}^{2}+3}} \\
& +\frac{y_{r}\left(\lambda_{V}^{4}-12 \lambda_{V}^{2}+3 \sqrt{24-7 \lambda_{V}^{2}} \lambda_{V}+\sqrt{24-7 \lambda_{V}^{2}} \lambda_{V}^{3}-9\right)}{2 \lambda_{V} \sqrt{24-7 \lambda_{V}^{2}}\left(\lambda_{V}^{2}+3\right)} \\
& -\frac{3 \sqrt{\frac{3}{2}} z_{r}\left(\sqrt{24-7 \lambda_{V}^{2}}-3 \lambda_{V}\right)\left(2 \lambda_{V}^{4}-3 \lambda_{V}^{2}+9\right)}{8 \lambda_{V} \sqrt{24-7 \lambda_{V}^{2}}\left(\lambda_{V}^{2}+3\right)\left(2 \lambda_{V}^{2}-3\right)}
\end{aligned}
$$


we find that the local invariant center manifold of $G$ is given by the graph

$$
\begin{aligned}
& \left\{\left(u, v_{1}, v_{2}, v_{3}\right) \mid v_{1}=\mathcal{O}(4),\right. \\
& v_{2}=-\frac{\sqrt{\frac{3}{2}} u^{2}\left[\lambda_{V}\left(3 \lambda_{V}^{2}-\sqrt{24-7 \lambda_{V}^{2}} \lambda_{V}-15\right)-3 \sqrt{24-7 \lambda_{V}^{2}}\right]}{2 \lambda_{V} \sqrt{\lambda_{V}^{2}+3}\left(7 \lambda_{V}^{2}-\sqrt{24-7 \lambda_{V}^{2}} \lambda_{V}-24\right)}+\mathcal{O}(4), \\
& \left.v_{3}=\frac{\sqrt{\frac{3}{2}} u^{2}\left[\lambda_{V}\left(-3 \lambda_{V}^{2}-\sqrt{24-7 \lambda_{V}^{2}} \lambda_{V}+15\right)-3 \sqrt{24-7 \lambda_{V}^{2}}\right]}{2 \lambda_{V} \sqrt{\lambda_{V}^{2}+3}\left(7 \lambda_{V}^{2}+\sqrt{24-7 \lambda_{V}^{2}} \lambda_{V}-24\right)}+\mathcal{O}(4),|u|<\delta\right\},
\end{aligned}
$$

with $\delta>0$ a suitably small positive real number. The dynamics at the center manifold is given by

$$
u^{\prime}=\frac{3 u^{2} \sqrt{\lambda_{V}^{2}+3}}{\lambda_{V}^{2}},|u|<\delta,
$$

with solution for $u(0)=u_{0}$ given by

$$
u(\tau)=\frac{u_{0} \lambda_{V}^{2}}{\lambda_{V}^{2}-3 u_{0} \sqrt{\lambda_{V}^{2}+3} \tau} .
$$

Since the origin is an inflection point of the potential $U(u)=-\frac{u^{3} \sqrt{\lambda_{V}^{2}+3}}{\lambda_{V}^{2}}$ of the gradient-like equation (B.10), it follows that $G$ is a saddle point for either $3<\lambda_{V}^{2}<\frac{24}{7}$ or $\lambda_{V}^{2}>\frac{24}{7}$, only for perturbations in the direction of $v_{r}$-axis and thus for perturbations in the $v$-axis.

\section{B.2 Stability of the critical points at infinity}

We consider the Poincaré variables (B.1) (we restrict the angle $\psi$ to this interval since the physical region is given by $y>0)$, and thus the points at infinity $(r \rightarrow+\infty)$ are those having $\rho \rightarrow 1$. Furthermore, the physical phase space is given by

$$
\left(x_{r}, y_{r}, z_{r}, v_{r}\right) \in[-1,1] \times[0,1] \times[-1,1] \times[-1,1]
$$

such that

$$
\frac{x_{r}^{2}+y_{r}^{2}}{1-v_{r}^{2}-x_{r}^{2}-y_{r}^{2}-z_{r}^{2}}-\frac{x_{r}^{2} z_{r}\left(\sqrt{6} n v_{r} x_{r}-6 v_{r}^{2}-6 x_{r}^{2}-6 y_{r}^{2}-6 z_{r}^{2}+6\right)}{\left(1-v_{r}^{2}-x_{r}^{2}-y_{r}^{2}-z_{r}^{2}\right)^{5 / 2}} \leq 1,
$$

and $v_{r}^{2}+x_{r}^{2}+y_{r}^{2}+z_{r}^{2} \leq 1$.

Performing the transformation (B.1) on the system (3.24)-(3.27), and taking the limit $\rho \rightarrow 1$, the leading terms in the resulting system are

$$
\begin{aligned}
& \left(1-\rho^{2}\right) \rho^{\prime} \rightarrow-\frac{1}{2} n(n+1) \cos ^{2} \theta \sin ^{2} \Phi \cos ^{2} \Phi \sin ^{4} \psi\left[2 \cos (2 \theta) \sin ^{2} \Phi \sin ^{2} \psi+\cos 2 \psi+1\right] \\
& \left(1-\rho^{2}\right)^{2} \theta^{\prime} \rightarrow-\frac{1}{2} n(n+1) \sin \theta \cos ^{3} \theta \sin ^{2}(2 \Phi) \sin ^{4} \psi \\
& \left(1-\rho^{2}\right)^{2} \Phi^{\prime} \rightarrow n(n+1) \cos ^{2} \theta \sin ^{2} \Phi \cos ^{2} \Phi \sin ^{5} \psi \cos \psi\left[\cos (2 \theta) \sin ^{2} \Phi-1\right], \\
& \left(1-\rho^{2}\right)^{2} \psi^{\prime} \rightarrow n(n+1) \cos ^{2} \theta \cos (2 \theta) \sin ^{3} \Phi \cos ^{3} \Phi \sin ^{4} \psi
\end{aligned}
$$


Since the equation for $\rho$ decouples, we only need to investigate the subsystem of the angular variables. Thus, equating (B.14), (B.15) and (B.16) to zero we obtain four classes of solutions:

\section{Class A:}

$$
x_{r}=0, y_{r}=\cos \psi, z_{r}= \pm \sin \Phi \sin \psi, v_{r}=\cos \Phi \sin \psi,
$$

for some $\Phi, \psi$. These points correspond to $\theta= \pm \frac{\pi}{2}$. For these points, $\Omega_{D E}$ of (3.36) diverges as $\rho \rightarrow 1^{-}$, unless $y_{r}=0$, thus the meaningful critical points at infinity are those contained in the invariant circle $z_{r}^{2}+v_{r}^{2}=1$. Representative critical points of this curve are the points

$$
L^{ \pm}:\left[x_{r}=0, y_{r}=0, z_{r}= \pm 1, v_{r}=0\right]
$$

and

$$
M^{ \pm}:\left[x_{r}=0, y_{r}=0, z_{r}=0, v_{r}= \pm 1\right]
$$

\section{Class B:}

$$
x_{r}=0, y_{r}=\cos \psi, z_{r}=0, v_{r}= \pm \sin \psi,
$$

for some $\psi$. These points correspond to $\Phi=0, \pi$, but since $\Omega_{D E}$ of (3.36) diverges as $\rho \rightarrow 1^{-}$unless $y_{r}=0$, all these points are unphysical apart from

$$
M^{ \pm}:\left[x_{r}=0, y_{r}=0, z_{r}=0, v_{r}= \pm 1\right] .
$$

\section{Class C:}

$$
x_{r}=0, y_{r}=1, z_{r}=0, v_{r}=0 .
$$

These points correspond to $\psi=0$. They lead to a divergence in $\Omega_{D E}$ in (3.36) and thus they are unphysical.

Class D:

$$
x_{r}= \pm \cos \theta \sin \psi, y_{r}=\cos \psi, z_{r}= \pm \sin \theta \sin \psi, v_{r}=0 .
$$

These points correspond to $\Phi= \pm \frac{\pi}{2}$, but since $\Omega_{D E}$ of (3.36) diverges unless $x_{r}=y_{r}=0$, all these points are unphysical apart from

$$
L^{ \pm}:\left[x_{r}=0, y_{r}=0, z_{r}= \pm 1, v_{r}=0\right] .
$$

¿From the above analysis we deduce that for $n \neq 0$ the singular points at infinity satisfy $x_{r}=0$ (we mention that $x_{r}=0$ is not an invariant set for the flow unless $y_{r}=0$ or $\lambda=0)$ that is points $M^{ \pm}$, or $v_{r}=0$ that is points $L^{ \pm}$.

Analyzing the dynamical system for $x_{r}, y_{r}, z_{r}, v_{r}$ around $M^{ \pm}$, we find that the eigenvalues of the linearization are $\left(0, \frac{3}{2},-\frac{3}{2}, \frac{9}{2}\right)$, and thus, although non-hyperbolic, points $M^{ \pm}$ behave as saddle points for the Poincaré vector field. 
In order to calculate the eigenvalues of the linearization of the dynamical system for $x_{r}, y_{r}, z_{r}, v_{r}$ around $L^{ \pm}$, we proceed as follows: having calculated the linear perturbation matrix $\mathbf{Q}$ we evaluate it at $y_{r}=v_{r}=0$, then we take the limit $x_{r} \rightarrow 0$ and finally we incorporate the lateral limit $z_{r} \rightarrow 1^{-}$(that is from below) for $L^{+}$or $z_{r} \rightarrow-1^{+}$(that is from above) for $L^{-}$. Calculating the eigenvalues of the resulting matrix we obtain that the eigenvalues of the linearization for both $L^{ \pm}$are given by $\left(3, \frac{15}{4}, \frac{9}{2}, \frac{9}{4}\right)$. Thus, we deduce that points $L^{ \pm}$are unstable.

\section{References}

[1] E. J. Copeland, M. Sami and S. Tsujikawa, Dynamics of dark energy, Int. J. Mod. Phys. D 15, 1753 (2006) [arXiv:hep-th/0603057].

[2] S. 'i. Nojiri and S. D. Odintsov, Introduction to modified gravity and gravitational alternative for dark energy, eConf C 0602061, 06 (2006) [Int. J. Geom. Meth. Mod. Phys. 4, 115 (2007)] [arXiv:hep-th/0601213].

[3] S. Capozziello and M. De Laurentis, Extended Theories of Gravity, Phys. Rept. 509, 167 (2011) [arXiv:1108.6266].

[4] M. Ostrogradsky, Mem. Acad. St. Petersbourg VI 4, 385 (1850).

[5] G. W. Horndeski, Second-order scalar-tensor field equations in a four-dimensional space, Int. J. Theor. Phys. 10, 363-384 (1974).

[6] A. Nicolis, R. Rattazzi and E. Trincherini, The Galileon as a local modification of gravity, Phys. Rev. D 79, 064036 (2009) [arXiv:0811.2197].

[7] C. Deffayet, G. Esposito-Farese, and A. Vikman, Covariant Galileon, Phys. Rev. D 79, 084003 (2009) [arXiv: 0901.1314].

[8] C. Deffayet, S. Deser, and G. Esposito-Farese, Generalized Galileons: All scalar models whose curved background extensions maintain second-order field equations and stress-tensors, Phys. Rev. D 80, 064015 (2009) [arXiv:0906.1967].

[9] C. Deffayet, X. Gao, D. A. Steer and G. Zahariade, From k-essence to generalised Galileons, Phys. Rev. D 84, 064039 (2011) [arXiv:1103.3260].

[10] A. Nicolis, R. Rattazzi and E. Trincherini, Energy's and amplitudes' positivity, JHEP 1005, 095 (2010) [Erratum-ibid. 1111, 128 (2011)], [arXiv: 0912.4258].

[11] C. Deffayet, O. Pujolas, I. Sawicki and A. Vikman, Imperfect Dark Energy from Kinetic Gravity Braiding, JCAP 1010, 026 (2010) [arXiv: 1008.0048].

[12] C. Germani, L. Martucci and P. Moyassari, Introducing the Slotheon: a slow Galileon scalar field in curved space-time, Phys. Rev. D 85, 103501 (2012) [arXiv:1108.1406].

[13] K. Hinterbichler, M. Trodden and D. Wesley, Multi-field galileons and higher co-dimension branes, Phys. Rev. D 82, 124018 (2010) [arXiv: 1008.1305].

[14] A. I. Vainshtein, To the problem of nonvanishing gravitation mass, Phys. Lett. B 39, 393 (1972).

[15] A. De Felice, R. Kase and S. Tsujikawa, Vainshtein mechanism in second-order scalar-tensor theories, Phys. Rev. D 85, 044059 (2012) [arXiv:1111.5090]. 
[16] N. Chow and J. Khoury, Galileon Cosmology, Phys. Rev. D 80, 024037 (2009) [arXiv:0905.1325].

[17] E. Babichev, C. Deffayet and G. Esposito-Farese, Improving relativistic MOND with Galileon $k$-mouflage, Phys. Rev. D 84, 061502 (2011) [arXiv:1106.2538].

[18] A. De Felice and S. Tsujikawa, Conditions for the cosmological viability of the most general scalar-tensor theories and their applications to extended Galileon dark energy models, JCAP 1202, 007 (2012) [arXiv:1110.3878].

[19] F. P. Silva and K. Koyama, Self-Accelerating Universe in Galileon Cosmology, Phys. Rev. D 80, 121301 (2009) [arXiv: 0909.4538].

[20] A. De Felice and S. Tsujikawa, Cosmology of a covariant Galileon field, Phys. Rev. Lett. 105, 111301 (2010) [arXiv: 1007.2700].

[21] N. Shirai, K. Bamba, S. Kumekawa, J. Matsumoto and S. 'i. Nojiri, Generalized Galileon Model - cosmological reconstruction and the Vainshtein mechanism -, Phys. Rev. D 86, 043006 (2012) [arXiv:1203.4962].

[22] Sampurnanand and A. ASen, DBI Galileon and Late time acceleration of the universe, JCAP 1212, 019 (2012) [arXiv:1208.0179].

[23] R. Gannouji and M. Sami, Galileon gravity and its relevance to late time cosmic acceleration, Phys. Rev. D 82, 024011 (2010) [arXiv: 1004.2808].

[24] D. F. Mota, M. Sandstad and T. Zlosnik, Cosmology of the selfaccelerating third order Galileon, JHEP 1012, 051 (2010) [arXiv: 1009.6151].

[25] P. Tretyakov, Scaling solutions in Galileon cosmology, Grav. Cosmol. 18, 93 (2012).

[26] C. Germani, On the Covariant Galileon and a consistent self-accelerating Universe, [arXiv: 1207.6414].

[27] S. Zabat and K. Nouicer, Phase space dynamics of the Galileon in modified gravity, AIP Conf. Proc. 1444, 291 (2011).

[28] P. Creminelli, A. Nicolis and E. Trincherini, Galilean Genesis: An Alternative to inflation, JCAP 1011, 021 (2010) [arXiv: 1007.0027].

[29] T. Kobayashi, M. Yamaguchi and J. 'i. Yokoyama, G-inflation: Inflation driven by the Galileon field,' Phys. Rev. Lett. 105, 231302 (2010) [arXiv:1008.0603].

[30] C. Burrage, C. de Rham, D. Seery and A. J. Tolley, Galileon inflation, JCAP 1101, 014 (2011) [arXiv: 1009. 2497].

[31] Z. -G. Liu, J. Zhang and Y. -S. Piao, A Galileon Design of Slow Expansion, Phys. Rev. D 84, 063508 (2011) [arXiv:1105.5713].

[32] T. Kobayashi, M. Yamaguchi and J. 'i. Yokoyama, Generalized G-inflation: Inflation with the most general second-order field equations, Prog. Theor. Phys. 126, 511 (2011) [arXiv: 1105.5723].

[33] J. Ohashi and S. Tsujikawa, Potential-driven Galileon inflation, [arXiv:1207.4879].

[34] S. Choudhury and S. Pal, DBI Galileon inflation in background SUGRA, [arXiv:1208.4433].

[35] K. Hinterbichler, A. Joyce, J. Khoury and G. E. J. Miller, DBI Realizations of the Pseudo-Conformal Universe and Galilean Genesis Scenarios, [arXiv:1209.5742].

[36] S. Mizuno and K. Koyama, Primordial non-Gaussianity from the DBI Galileons, Phys. Rev. 
D 82, 103518 (2010) [arXiv: 1009.0677].

[37] S. Renaux-Petel, Orthogonal non-Gaussianities from Dirac-Born-Infeld Galileon inflation, Class. Quant. Grav. 28, 182001 (2011) [Erratum-ibid. 28, 249601 (2011)], [arXiv:1105.6366].

[38] X. Gao and D. A. Steer, Inflation and primordial non-Gaussianities of 'generalized Galileons', JCAP 1112, 019 (2011) [arXiv:1107.2642].

[39] S. Renaux-Petel, S. Mizuno and K. Koyama, Primordial fluctuations and non-Gaussianities from multifield DBI Galileon inflation, JCAP 1111, 042 (2011) [arXiv:1108.0305].

[40] S. Choudhury and S. Pal, Large non-Gaussianities from DBI Galileon and resolution of sensitivity problem, [arXiv:1210.4478].

[41] L. Levasseur Perreault, R. Brandenberger and A. -C. Davis, Defrosting in an Emergent Galileon Cosmology, Phys. Rev. D 84, 103512 (2011) [arXiv:1105.5649].

[42] T. Kobayashi, Cosmic expansion and growth histories in Galileon scalar-tensor models of dark energy, Phys. Rev. D 81, 103533 (2010) [arXiv: 1003.3281].

[43] K. Hirano, Z. Komiya and H. Shirai, Constraining Galileon gravity from observational data with growth rate, Prog. Theor. Phys. 127, 1041 (2012) [arXiv:1103.6133].

[44] E. Bellini, N. Bartolo and S. Matarrese, Spherical Collapse in covariant Galileon theory, JCAP 1206, 019 (2012) [arXiv: 1202.2712].

[45] T. Qiu, J. Evslin, Y. -F. Cai, M. Li and X. Zhang, Bouncing Galileon Cosmologies, JCAP 1110, 036 (2011) [arXiv:1108.0593].

[46] D. A. Easson, I. Sawicki and A. Vikman, G-Bounce, JCAP 1111, 021 (2011) [arXiv: 1109.1047].

[47] Y. -F. Cai, D. A. Easson and R. Brandenberger, Towards a Nonsingular Bouncing Cosmology, JCAP 1208, 020 (2012) [arXiv: 1206.2382].

[48] T. Kobayashi, H. Tashiro and D. Suzuki, Evolution of linear cosmological perturbations and its observational implications in Galileon-type modified gravity, Phys. Rev. D 81, 063513 (2010) [arXiv: 0912 .4641].

[49] A. De Felice, R. Kase and S. Tsujikawa, Matter perturbations in Galileon cosmology, Phys. Rev. D 83, 043515 (2011) [arXiv: 1011.6132].

[50] A. Barreira, B. Li, C. Baugh and S. Pascoli, Linear perturbations in Galileon gravity models, Phys. Rev. D 86, 124016 (2012) [arXiv:1208.0600].

[51] X. Gao, Conserved cosmological perturbation in Galileon models, JCAP 1110 (2011) 021 [arXiv: 1106.0292].

[52] Z. -G. Liu and Y. -S. Piao, A Galileon Design of Slow Expansion: II, Phys. Lett. B 718, 734 (2013) [arXiv: 1207. 2568].

[53] L. Hui and A. Nicolis, A no-hair theorem for the galileon, [arXiv:1202.1296].

[54] S. Deser and J. Franklin, Symmetrically reduced Galileon equations and solutions, Phys. Rev. D 86, 047701 (2012) [arXiv: 1206.3217].

[55] M. Rinaldi, Galileon Black Holes, [arXiv: 1208.0103].

[56] T. L. Curtright, Galileons and Naked Singularities, Phys. Lett. B 716, 366 (2012) [arXiv: 1208.1205]. 
[57] G. L. Goon, K. Hinterbichler and M. Trodden, Stability and superluminality of spherical DBI galileon solutions, Phys. Rev. D 83, 085015 (2011) [arXiv: 1008.4580].

[58] E. Babichev, Galileon accretion, Phys. Rev. D 83, 024008 (2011) [arXiv:1009. 2921].

[59] M. Andrews, K. Hinterbichler, J. Khoury and M. Trodden, Instabilities of Spherical Solutions with Multiple Galileons and SO(N) Symmetry, Phys. Rev. D 83, 044042 (2011) [arXiv: 1008.4128].

[60] S. Endlich and J. Wang, Classical Stability of the Galileon, JHEP 1111, 065 (2011) [arXiv:1106.1659].

[61] J. Evslin, Stability of Closed Timelike Curves in a Galileon Model, JHEP 1203, 009 (2012) [arXiv:1112.1349].

[62] S. A. Appleby and E. V. Linder, The Paths of Gravity in Galileon Cosmology, JCAP 1203, 043 (2012) [arXiv:1112.1981 [astro-ph.CO]].

[63] A. Masoumi and X. Xiao, Moving Stable Solitons in Galileon Theory, [arXiv:1201.3132].

[64] S. -Y. Zhou, Note on the Stabilities of the Light-like Galileon Solutions, [arXiv:1202.5769].

[65] C. Burrage and D. Seery, Revisiting fifth forces in the Galileon model, JCAP 1008, 011 (2010) [arXiv: 1005.1927].

[66] A. Ali, R. Gannouji and M. Sami, Modified gravity a la Galileon: Late time cosmic acceleration and observational constraints, Phys. Rev. D 82, 103015 (2010) [arXiv: 1008.1588].

[67] S. Nesseris, A. De Felice and S. Tsujikawa, Observational constraints on Galileon cosmology, Phys. Rev. D 82, 124054 (2010) [arXiv: 1010.0407].

[68] S. A. Appleby and E. V. Linder, Trial of Galileon gravity by cosmological expansion and growth observations, JCAP 1208, 026 (2012) [arXiv:1204.4314].

[69] A. De Felice and S. Tsujikawa, Cosmological constraints on extended Galileon models, JCAP 1203, 025 (2012) [arXiv:1112.1774].

[70] M. .W. Hossain and A. A. Sen, Do Observations Favour Galileon Over Quintessence?, Phys. Lett. B 713, 140 (2012) [arXiv:1201.6192].

[71] H. Okada, T. Totani and S. Tsujikawa, Constraints on $f(R)$ theory and Galileons from the latest data of galaxy redshift surveys, [arXiv: 1208.4681].

[72] L. Iorio, Constraints on Galileon-induced precessions from solar system orbital motions, JCAP 1207, 001 (2012) [arXiv:1204.0745].

[73] P. Brax, C. Burrage and A. -C. Davis, Laboratory Tests of the Galileon, JCAP 1109, 020 (2011) [arXiv: 1106.1573].

[74] C. Deffayet, S. Deser and G. Esposito-Farese, Arbitrary p-form Galileons, Phys. Rev. D 82, 061501 (2010) [arXiv:1007.5278].

[75] A. Padilla, P. M. Saffin and S. -Y. Zhou, Bi-galileon theory I: Motivation and formulation, JHEP 1012, 031 (2010) [arXiv: 1007.5424].

[76] A. Padilla, P. M. Saffin and S. -Y. Zhou, Multi-galileons, solitons and Derrick's theorem, Phys. Rev. D 83, 045009 (2011) [arXiv: 1008.0745].

[77] A. Padilla, P. M. Saffin and S. -Y. Zhou, Bi-galileon theory II: Phenomenology, JHEP 1101, 099 (2011) [arXiv: 1008.3312]. 
[78] A. De Felice and S. Tsujikawa, Generalized Galileon cosmology, Phys. Rev. D 84, 124029 (2011) [arXiv: 1008.4236].

[79] S. -Y. Zhou, Goldstone's Theorem and Hamiltonian of Multi-galileon Modified Gravity, Phys. Rev. D 83, 064005 (2011) [arXiv: 1011.0863].

[80] K. Van Acoleyen and J. Van Doorsselaere, Galileons from Lovelock actions, Phys. Rev. D 83, 084025 (2011) [arXiv: 1102.0487].

[81] J. Khoury, J. -L. Lehners and B. A. Ovrut, Supersymmetric Galileons, Phys. Rev. D 84, 043521 (2011) [arXiv:1103.0003].

[82] C. Burrage, C. de Rham and L. Heisenberg, de Sitter Galileon, JCAP 1105, 025 (2011) [arXiv:1104.0155].

[83] M. Trodden and K. Hinterbichler, Generalizing Galileons, Class. Quant. Grav. 28, 204003 (2011) [arXiv: 1104.2088].

[84] J. Evslin and T. Qiu, Closed Timelike Curves in the Galileon Model, JHEP 1111, 032 (2011) [arXiv: 1106.0570].

[85] N. Kaloper, A. Padilla and N. Tanahashi, Galileon Hairs of Dyson Spheres, Vainshtein's Coiffure and Hirsute Bubbles, JHEP 1110, 148 (2011) [arXiv:1106.4827].

[86] V. Sivanesan, Hamiltonian of galileon field theory, [arXiv:1111.3558].

[87] S. -Y. Zhou and E. J. Copeland, Galileons with Gauge Symmetries, Phys. Rev. D 85, 065002 (2012) [arXiv:1112.0968].

[88] T. LCurtright and D. BFairlie, Geons of Galileons, Phys. Lett. B 716, 356 (2012) [arXiv: 1206.3616].

[89] T. d. P. Netto and I. L. Shapiro, One-loop divergences in the Galileon model, Phys. Lett. B 716, 454 (2012) [arXiv: 1207.0534].

[90] A. Ali, R. Gannouji, M. .W. Hossain and M. Sami, Light mass galileons: Cosmological dynamics, mass screening and observational constraints, [arXiv:1207.3959].

[91] E. Babichev, Plane waves in the generalized Galileon theory, [arXiv:1207.4764].

[92] P. Creminelli, K. Hinterbichler, J. Khoury, A. Nicolis and E. Trincherini, Subluminal Galilean Genesis, [arXiv: 1209.3768].

[93] A. Padilla and V. Sivanesan, Covariant multi-galileons and their generalisation, [arXiv: 1210.4026].

[94] Y. -Z. Chu and M. Trodden, Retarded Green's Function Of A Vainshtein System And Galileon Waves, [arXiv:1210.6651].

[95] C. de Rham and A. J. Tolley, DBI and the Galileon reunited, JCAP 1005, 015 (2010) [arXiv: 1003.5917].

[96] C. de Rham and L. Heisenberg, Cosmology of the Galileon from Massive Gravity, Phys. Rev. D 84, 043503 (2011) [arXiv: 1106.3312].

[97] G. Goon, K. Hinterbichler and M. Trodden, Symmetries for Galileons and DBI scalars on curved space, JCAP 1107, 017 (2011) [arXiv: 1103.5745].

[98] R. Gannouji and M. Sami, Vainshtein mechanism in Gauss-Bonnet gravity and Galileon aether, Phys. Rev. D 85, 024019 (2012) [arXiv:1107.1892]. 
[99] G. Goon, K. Hinterbichler and M. Trodden, Galileons on Cosmological Backgrounds, JCAP 1112, 004 (2011) [arXiv: 1109.3450].

[100] C. Burrage, C. de Rham, L. Heisenberg and A. J. Tolley, Chronology Protection in Galileon Models and Massive Gravity, [arXiv:1111.5549].

[101] G. Goon, K. Hinterbichler, A. Joyce and M. Trodden, Gauged Galileons From Branes, [arXiv: 1201.0015$]$.

[102] G. Goon, K. Hinterbichler, A. Joyce and M. Trodden, Galileons as Wess-Zumino Terms, [arXiv: 1203.3191].

[103] C. de Rham, Galileons in the Sky, Comptes Rendus Physique 13, 666 (2012) [arXiv:1204.5492].

[104] C. Charmousis, B. Gouteraux and E. Kiritsis, Higher-derivative scalar-vector-tensor theories: black holes, Galileons, singularity cloaking and holography, JHEP 1209, 011 (2012) [arXiv: 1206.1499].

[105] G. Gabadadze, K. Hinterbichler, J. Khoury, D. Pirtskhalava and M. Trodden, A Covariant Master Theory for Novel Galilean Invariant Models and Massive Gravity, [arXiv: 1208.5773].

[106] M. Zumalacarregui, T. S. Koivisto and D. F. Mota, DBI Galileons in the Einstein Frame: Local Gravity and Cosmology, [arXiv:1210.8016].

[107] E. J. Copeland, A. R. Liddle and D. Wands, Exponential potentials and cosmological scaling solutions, Phys. Rev. D 57, 4686 (1998) [arXiv:gr-qc/9711068].

[108] P. G. Ferreira and M. Joyce, Structure formation with a self-tuning scalar field, Phys. Rev. Lett. 79, 4740 (1997) [arXiv: astro-ph/9707286].

[109] Y. Gong, A. Wang and Y. Z. Zhang, Exact scaling solutions and fixed points for general scalar field, Phys. Lett. B 636, 286 (2006) [arXiv:gr-qc/0603050].

[110] X. m. Chen, Y. g. Gong and E. N. Saridakis, Phase-space analysis of interacting phantom cosmology, JCAP 0904, 001 (2009) [arXiv:0812.1117].

[111] G. Leon and E. N. Saridakis, Phase-space analysis of Horava-Lifshitz cosmology, JCAP 0911, 006 (2009) [arXiv:0909.3571].

[112] H. -J. Schmidt, New exact solutions for power law inflation Friedmann models, Astron. Nachr. 311, 165 (1990) [arXiv:gr-qc/0109004].

[113] V. Muller, H. J. Schmidt and A. A. Starobinsky, Power law inflation as an attractor solution for inhomogeneous cosmological models, Class. Quant. Grav. 7, 1163 (1990).

[114] R. J. Scherrer and A. A. Sen, Thawing quintessence with a nearly flat potential, Phys. Rev. D 77, 083515 (2008) [arXiv:0712.3450].

[115] R. J. Scherrer and A. A. Sen, Phantom Dark Energy Models with a Nearly Flat Potential, Phys. Rev. D 78, 067303 (2008) [arXiv:0808.1880].

[116] M. R. Setare and E. N. Saridakis, Quintom dark energy models with nearly flat potentials, Phys. Rev. D 79, 043005 (2009) [arXiv:0810.4775].

[117] S., Lynch, Dynamical Systems with Applications using Mathematica, Birkhauser, Boston (2007).

[118] G. Leon, On the Past Asymptotic Dynamics of Non-minimally Coupled Dark Energy, Class. 
Quant. Grav. 26, 035008 (2009) [arXiv:0812.1013].

[119] C. Xu, E. N. Saridakis and G. Leon, Phase-Space analysis of Teleparallel Dark Energy, JCAP 1207, 005 (2012) [arXiv: 1202.3781].

[120] G. Leon and C. R. Fadragas, Cosmological Dynamical Systems, LAP LAMBERT Academic Publishing, (2011).

[121] A. De Felice and T. Tanaka, Inevitable ghost and the degrees of freedom in $f(R, G)$ gravity, Prog. Theor. Phys. 124, 503 (2010) [arXiv:1006.4399].

[122] A. De Felice, T. Suyama and T. Tanaka, Stability of Schwarzschild-like solutions in $f(R, G)$ gravity models, Phys. Rev. D 83, 104035 (2011) [arXiv:1102.1521].

[123] B. Aulbach, Continuous and discrete dynamics near manifolds of equilibria, Lecture Notes in Mathematics, No 1058, Springer-Verlag (1981). 